\title{
Averaging Operations for Lattice Gauge Theories *
}

\author{
T. Bałaban \\ Department of Physics, Harvard University, Cambridge, MA 02138, USA
}

\begin{abstract}
Usually renormalization group transformations are defined by some averaging operations. In this paper we study such operations for lattice gauge fields and for gauge transformations. We are interested especially in characterizing some classes of field configurations on which the averaging operations are regular (e.g., analytic). These results will be used in subsequent papers on the renormalization group method in lattice gauge theories.
\end{abstract}

\section{Introduction}

In Wilson's approach to renormalization group transformations $[9,10]$ for lattice gauge systems, it is necessary to define an operation of taking an average of field configurations over subdomains of a lattice. These subdomains are usually some simple subsets, for example cubes of a fixed size, or sums of several such cubes. In this paper we will study one such definition of an averaging operation. This operation will be used in other papers on gauge field theories.

Let us introduce some definitions and notations. We will be very sketchy because these definitions have already appeared several times in the earlier papers $[1,2]$ of the author and we refer the reader to these papers, especially to [2], for more detailed explanations. We consider a subdomain $\Omega$ of the lattice $\eta Z^{d}$ with a lattice spacing $\eta$. A sequence of sets $\Omega^{(j)}$ is defined as the intersections

$$
\Omega^{(j)}=\Omega \cap L^{j} \eta Z^{d},
$$

where $L$ is a fixed integer, $L>1$. For a point $y \in L^{n} \eta Z^{d}$ (or any lattice $\delta Z^{d}$ ), we define a block of an order $j$ as the cube

$$
B^{j}(y)=\left\{x \in L^{-j} L^{n} \eta Z^{d}: y_{\mu} \leqq x_{\mu}<y_{\mu}+L^{n} \eta, \mu=1, \ldots, d\right\}
$$

(or the corresponding cube with $L^{n} \eta$ replaced by $\delta$ ). We will omit the subscript $j$ if $j=1$. For a subset $\Lambda \subset L^{n} \eta Z^{d}$ (or $\left.\subset \delta Z^{d}\right)$, we define

$$
B^{j}(\Lambda)=\bigcup_{y \in A} B^{j}(y) \subset L^{-j} L^{n} \eta Z^{d} \text { (or } C L^{-j} \delta Z^{d} \text { ). }
$$

* $\quad$ Research supported in part by the National Science Foundation under Grant PHY-82-03669 
We assume that

$$
B^{j}\left(\Omega^{(j)}\right)=\Omega \quad \text { for } j=1, \ldots, k
$$

for some $k$. In fact, we will assume that $\eta=L^{-k}$. Thus $\Omega$ is a sum of blocks of the order $k$.

Besides the blocks of different orders, there are two other geometric objects important for us. Bonds of the lattice $\Omega$ are ordered pairs $\left\langle x, x^{\prime}\right\rangle$ of nearest neighbor points $x, x^{\prime}$ of $\Omega$. We identify them with the corresponding oriented intervals with endpoints $x, x^{\prime}$. We will denote them also by the letters $b, b^{\prime}, c$, etc. For example, $b$ denotes a bond $\left\langle b_{-}, b_{+}\right\rangle$with an initial point $b_{-}$and a final point $b_{+} ; b_{-}, b_{+}$are nearest neighbors. Plaquettes are oriented elementary squares of the lattice $\Omega$ and are denoted by $p, p^{\prime}$, etc. A boundary of a plaquette $p$ is a sum of four bonds, and an orientation of the plaquette may be indicated by an orientation of the bonds. A plaquette $p$ may be identified also with an ordered quadruple $\langle x, y, z, w\rangle$ of corners $x, y, z, w$ of the elementary square $p$; the ordering indicates the orientations of bonds $\langle x, y\rangle,\langle y, z\rangle,\langle z, w\rangle,\langle w, x\rangle$ forming the boundary $\partial p$. Speaking precisely, the symbol $\langle x, y, z, w\rangle$ indicates not only the oriented square, but also the initial point $x$ of its boundary. In this paper we will consider positively oriented plaquettes and such a plaquette $p \subset \Omega$ is represented as

$$
p=\left\langle x, x+\eta e_{\mu}, x+\eta e_{\mu}+\eta e_{v}, x+\eta e_{v}\right\rangle, \quad \mu<v .
$$

The above definitions of bonds and plaquettes may be applied to an arbitrary lattice, e.g., to $\Omega^{(j)}$.

Gauge field configurations $U$ are defined on a set of bonds in $\Omega$, and with values in a Lie subgroup $G$ of a unitary group $U(N)$. A value of $U$ at a bond $b=\left\langle x, x^{\prime}\right\rangle$ is denoted by

$$
U_{b}=U_{\left\langle x, x^{\prime}\right\rangle}=U\left(x, x^{\prime}\right)=U(b),
$$

and we assume that $U$ satisfies the condition

$$
U\left(x, x^{\prime}\right)=U^{-1}\left(x^{\prime}, x\right)=U^{*}\left(x^{\prime}, x\right) .
$$

In the sequel, we will consider quantities which are invariant with respect to some important transformations in the space of gauge field configurations, the so-called gauge transformations. They are determined by gauge functions $u: \Omega \rightarrow G$, and are given by

$$
U^{u}\left(x, x^{\prime}\right)=u(x) U\left(x, x^{\prime}\right) u^{-1}\left(x^{\prime}\right), \quad\left\langle x, x^{\prime}\right\rangle \subset \Omega
$$

For an oriented contour $\Gamma=\bigcup_{i=0}^{n-1}\left\langle x_{i}, x_{i+1}\right\rangle$ we define

$$
U(\Gamma)=\prod_{i=0}^{n-1} U\left(x_{i}, x_{i+1}\right)
$$

where the order of factors in the product is the same as the order of bonds in $\Gamma$. This definition will be applied also to contours on arbitrary lattices. For a plaquette $p=\langle x, y, z, w\rangle$ we define $\partial p$ as the oriented contour $\partial p=\langle x, y\rangle \cup\langle y, z\rangle \cup\langle z, w\rangle$ $\cup\langle w, x\rangle$, and $U(\partial p)$ is defined by (9). 
The renormalization group transformations are integral operators transforming functions defined on gauge field configurations on a lattice, into functions defined on configurations on the lattice of blocks. We will consider transformations of the form

$$
\varrho^{\prime}(V)=\int d U \delta\left(V \bar{U}^{-1}\right) \varrho(U),
$$

where, for example, $U$ is a gauge field configuration on the lattice $\Omega^{(j)}, V$ is a configuration on the lattice $\Omega^{(j+1)}$ and $d U$ is a product of Haar measures of the group $G$. The most important part of the above definition is an averaging operation $\bar{U}$. It transforms a configuration $U$ defined on $\Omega^{(j)}$ into a configuration $\bar{U}$ defined on $\Omega^{(j+1)}$. In fact, $\Omega^{(j)}$ may be replaced by any other lattice. We will demand that this averaging operation has to satisfy some important and natural conditions. A first condition is connected with the fact that we consider gaugeinvariant quantities, so we demand that the averaging preserves gauge transformations, i.e.,

$$
\left(\overline{U^{u}}\right)\left(y, y^{\prime}\right)=u(y) \bar{U}\left(y, y^{\prime}\right) u^{-1}\left(y^{\prime}\right), \quad \text { or } \quad \overline{U^{u}}=(\bar{U})^{u} .
$$

This property implies that the renormalization transformation (10) transforms gauge-invariant functions $\varrho$ into gauge-invariant $\varrho^{\prime}$. Indeed, if $v$ is a gauge function on a new lattice, then

$$
\begin{aligned}
\varrho^{\prime}\left(V^{v}\right) & =\int d U \delta\left(V^{v} \bar{U}^{-1}\right) \varrho(U)=\int d U \delta\left(V^{v}\left(\overline{U^{u}}\right)^{-1}\right) \varrho\left(U^{u}\right) \\
& =\int d U \delta\left(V^{v}\left(\bar{U}^{u}\right)^{-1}\right) \varrho(U),
\end{aligned}
$$

where we have used the gauge-invariance of the Haar measure $d U$ and the function $\varrho$, besides the condition (11). Now if we choose $u$ coinciding with $v$ at points of the new lattice, then we get

$$
\begin{aligned}
\varrho^{\prime}\left(V^{v}\right) & =\int d U \prod_{\left\langle y, y^{\prime}\right\rangle} \delta\left(v(y) V\left(y, y^{\prime}\right)\left(\bar{U}\left(y, y^{\prime}\right)\right)^{-1} v^{-1}(y)\right) \varrho(U) \\
& =\int d U \delta\left(V \bar{U}^{-1}\right) \varrho(U)=\varrho^{\prime}(V) .
\end{aligned}
$$

We have used also the invariance of the $\delta$-function concentrated at the identity of the group $G$ with respect to transformations $U \rightarrow v U v^{-1}$. A second condition on averages $\bar{U}$ is formulated in the following way. We consider configurations $U$ with values in a small neighborhood of the identity of $G$, hence $U=e^{i A}$ and $A$ is a Lie algebra valued configuration with values in a small neighborhood of 0 . For such configurations we demand that $\frac{1}{i} \log \bar{U}$ is well approximated by the linear averaging operation (1.8) defined in [2]. Let us write this operation

$$
\bar{A}_{c}=\sum_{x \in B\left(c_{-}\right)} L^{-(d+1)}\left(A\left(\Gamma_{c_{-}, x}\right)+A([x, x(c)])+A\left(\Gamma_{x(c), c_{+}}\right)\right) .
$$

We refer the reader to paper [2] for explanations of symbols used above. Let us notice that $\Gamma_{c_{-}, x} \cup[x, x(c)] \cup \Gamma_{x(c), c_{+}}$is an oriented contour with $c_{-}$as an initial point and $c_{+}$as a final point. We denote it by $\Gamma_{c, x}$.

There are many ways of implementing these two conditions. We will write now a definition of an average which satisfies these conditions and which has some other advantages. We define

$$
\bar{U}_{c}=\exp \left[i \sum_{x \in B\left(c_{-}\right)} L^{-d} \frac{1}{i} \log U\left(\Gamma_{c, x}\right) U(c)^{-1}\right] U(c) .
$$


It is easy to see that taking $U=e^{i A}$ with $A$ small and expanding the logarithm of the expression on the right-hand side above in powers of $A$, we get the expression (14) as a linear term in the expansion.

In this paper we will study properties of this and other averaging operations, and especially their compositions. In the future we will need many properties of these compositions. For example, it will be necessary to know that a composition of many averaging operations applied to a regular or small gauge field configuration gives a regular or small configuration also, the notions of regularity or smallness being related to scales on which the gauge field configurations are considered. This is the basic property of averagings we would like to understand. Another important property is an analyticity of a result of the averaging operation with respect to an averaged field.

The analysis and the propositions we prove in this paper can be easily extended to other definitions of averaging operations satisfying the requirements postulated in this introduction. The definitions we have used in this paper were chosen for their simplicity but in some future papers we will need somewhat more complicated definitions. We will discuss them at the proper time when they appear.

\section{A. Norms, Important Inequalities, and Functions}

We consider a Lie subgroup $G$ of a unitary group $U(N)$. Its Lie algebra $\mathfrak{g}$ is a subalgebra of the algebra of hermitian matrices. Because of analyticity properties we are looking for, we will consider complexified algebras and groups. The complexification of the algebra of hermitian matrices is the algebra of all complex $N \times N$ matrices, and the complexification of $\mathrm{U}(N)$ is the general linear complex group $\mathrm{GL}(\mathbb{C}, N)$.

The complexification $\mathfrak{g}^{c}$ is a subalgebra of the algebra of all complex matrices. It may be defined in the following way: we take a basis $\left\{t_{a}\right\}$ of the algebra $g$, then all elements $A \in \mathfrak{g}$ can be written as linear combinations $A=\sum_{a} A_{a} t_{a}$, where $A_{a}$ are arbitrary real numbers, $A_{a} \in \mathbb{R}$, and we form a complexification $\mathfrak{g}^{c}$ taking $A_{a}$ as arbitrary complex numbers, $A_{a} \in \mathbb{C}$. It is easy to see that this complexification is independent of any particular basis $\left\{t_{a}\right\}$ chosen in the above definition. For a function $F(A)$ defined on the complexified algebra, a notion of analyticity is well defined and means analyticity with respect to complex variables $A_{a}$. The unitary group $\mathrm{U}(N)$ can be obtained by an application of the exponential mapping to the algebra of hermitian matrices. This exponential mapping is simply given by the exponential function $e^{i A}$, where

$$
e^{X}=\exp X=\sum_{n=0}^{\infty} \frac{X^{n}}{n !}
$$

is defined for an arbitrary matrix $X$. The group $G$ is obtained by applying the function $e^{i A}$ to $A \in \mathfrak{g}$, and the complexified group $G^{c}$ may be defined as an image of the exponential function applied to the complexified algebra $\mathfrak{g}^{c}$. Of course, $G^{c}$ is a subgroup of $\mathrm{GL}(\mathbb{C}, N)$. We will need only a neighborhood of $G$ in this subgroup. We introduce a scalar product in the algebra of all complex matrices

$$
\langle X, Y\rangle=\operatorname{tr} X^{*} Y, \operatorname{tr} X=\frac{1}{N} \sum_{j=1}^{N} X_{j, j}
$$


and a scalar product in spaces of matrix valued functions defined on subsets $\Omega$ $\subset \eta Z^{d}$

$$
\langle X, Y\rangle=\sum_{x \in \Omega} \eta^{d} \operatorname{tr} X^{*}(x) Y(x),
$$

similarly for functions defined at bonds or plaquettes of $\Omega$. The scalar products define the corresponding norms. They are $L^{2}$ norms and are denoted by $\|\cdot\|$, e.g., for a matrix $X$ the norm is given by $\|X\|^{2}=\operatorname{tr} X^{*} X$. The $L^{p}$ norms, $1 \leqq p \leqq \infty$, are defined in an obvious way. In estimates we will use much more frequently another norm for matrices. It is the operator norm given by

$$
\begin{gathered}
|X|=\sup |X \psi|=\sup |\bar{\phi} \cdot X \psi|, \quad \phi, \psi \in \mathbb{C}^{N}, \\
|\phi|=|\psi|=1,|\psi|^{2}=\sum_{j=1}^{N}\left|\psi_{j}\right|^{2} .
\end{gathered}
$$

We have the following inequalities for the norms introduced:

$$
\begin{gathered}
|\operatorname{tr} X| \leqq|X|,\|X\| \leqq|X|,|X| \leqq \sqrt{N}\|X\|, \\
|X Y| \leqq|X||Y|,\|X Y\| \leqq\|X\|\|Y\| .
\end{gathered}
$$

Now we will introduce several important functions on the matrix algebra. The first is a logarithmic function. It is an inverse to the exponential function and for matrices $X$ satisfying $|X-1|<1$ it is given by

$$
\log X=\sum_{n=1}^{\infty} \frac{(-1)^{n+1}}{n}(X-1)^{n} .
$$

Of course, both functions are analytic functions of complex matrices $X$. For any branch $\log z$ of the ordinary $\log$ arithmic function, we may define $\log X$ for $X$ defining a normal operator on $\mathbb{C}^{N}$. If $X$ is such a matrix, then $X=\sum_{j=1}^{r} z_{j} P_{j}, z_{j} \in \mathbb{C}$, $\left\{P_{j}\right\}$ is a spectral family of orthogonal projections (i.e., $P_{j}^{*}=P_{j}, P_{j} P_{k}=P_{k} P_{j}$ $\left.=\delta_{j k} P_{j}\right)$, and we define

$$
\log X=\sum_{j=1}^{r} \log z_{j} P_{j}
$$

We will use this definition for $\log z=\log |z|+i \arg z$, where $\arg z \in]-\pi, \pi]$, and for unitary matrices. Every unitary matrix $U$ can be represented uniquely in the form $U=\sum_{j=1}^{r} e^{i \lambda_{j}} P_{j}$, where the numbers $\lambda_{j}$ are different and satisfy $\left.\left.\lambda_{j} \in\right]-\pi, \pi\right]$, and then we define

$$
\log U=i \sum_{j=1}^{r} \lambda_{j} P_{j}=i A,
$$

$A$ is a hermitian matrix, $|A| \leqq \pi$. From this definition the following inequalities follow:

$$
\begin{gathered}
|U-1|=\max _{j}\left|e^{i \lambda_{j}}-1\right|=\max _{j}\left|\frac{\sin \frac{\lambda_{j}}{2}}{\frac{\lambda_{j}}{2}}\right|\left|\lambda_{j}\right| \leqq \max _{j}\left|\lambda_{j}\right|=|\log U|, \\
|\log U| \leqq \frac{\pi}{2}|U-1|, \text { because }\left|\frac{\sin x}{x}\right| \geqq \frac{2}{\pi} \quad \text { for } \quad x \in\left[-\frac{\pi}{2}, \frac{\pi}{2}\right] .
\end{gathered}
$$


We will need also inequalities of this type for arbitrary complex matrices $X$ instead of $U$. For matrices $X$ satisfying $|X-1| \leqq \frac{1}{2}$, we have

$$
\begin{gathered}
|\log X| \leqq \sum_{n=1}^{\infty} \frac{1}{n}|X-1|^{n} \leqq \frac{|X-1|}{1-|X-1|} \leqq 2|X-1|, \\
|X-1|=\left|e^{\log X}-1\right| \leqq e^{|\log X|}-1 \leqq e^{|\log X|}|\log X| \leqq 2|\log X| .
\end{gathered}
$$

Let us consider now the most important function for all future considerations, the function describing the group multiplication in terms of Lie algebra elements. Let us define

$$
Z(u, v)=\log e^{u X} e^{v Y} .
$$

It is a well-defined and analytic function of $u X, v Y$, for example, in the domain $|u X|<\frac{1}{8},|v Y|<\frac{1}{8}$. Its power series expansion is given by the Baker-CampbellHaussdorf formula (see [7], Sect. 2.15)

$$
\begin{aligned}
Z= & \log e^{X} e^{Y}=\sum_{m=1}^{\infty} \sum_{p_{i}+q_{l} \geqq 1} \frac{(-1)^{m+1}}{m} \frac{1}{p_{1}+p_{2}+\ldots+p_{m}+q_{1}+q_{2}+\ldots+q_{m}} \\
& \cdot \frac{1}{p_{1} ! q_{1} ! \cdot \ldots \cdot p_{m} ! q_{m} !}\left(\operatorname{ad}_{X}\right)^{p_{1}}\left(\operatorname{ad}_{Y}\right)^{q_{1}} \cdot \ldots \cdot\left(\operatorname{ad}_{X}\right)^{p_{m}}\left(\operatorname{ad}_{Y}\right)^{q_{m}-1} Y \\
= & X+Y+\frac{1}{2}[X, Y]+\frac{1}{12}[Y,[Y, X]]+\frac{1}{12}[X,[X, Y]]+\ldots,
\end{aligned}
$$

where $\operatorname{ad}_{X} Y=[X, Y]=X Y-Y X$, and the series is convergent in a neighborhood of $0:|X|,|Y| \leqq c_{0}$ for some positive $c_{0}$. From the structure of this power series expansion, we get easily the bound

$$
\left|Z-X-Y-\frac{1}{2}[X, Y]\right| \leqq O(1)\left(|X|^{2}|Y|+|X||Y|^{2}\right),
$$

and this implies

$$
|Z-X-Y| \leqq 2|X||Y| \text { for }|X|,|Y| \leqq c_{1},
$$

where $c_{1}$ is a sufficiently small positive constant, $c_{1} \leqq c_{0}$. We will treat the expression $\operatorname{ad}_{X} Y$ for a fixed $X$ as a linear operator on a space of matrices $Y$, and we will consider functions of this operator $f\left(\operatorname{ad}_{X}\right)$. For analytic functions $f$ defined in a neighborhood of 0 and for $X$ with a sufficiently small norm, the function $f\left(\operatorname{ad}_{X}\right)$ can be defined by the power series expansion.

We will be interested in calculating derivatives of the function $Z(u, v)$ and some other functions of this type. Let us start with the following basic formula (see [7, Theorem 2.14.3])

$$
e^{-A(t)} \frac{d}{d t} e^{A(t)}=\sum_{n=0}^{\infty} \frac{(-1)^{n}}{(n+1) !}\left(\operatorname{ad}_{A(t)}\right)^{n} A^{\prime}(t)=g\left(\operatorname{ad}_{A(t)}\right) A^{\prime}(t),
$$

where $A(t)$ is a differentiable matrix-valued function of $t$. The function $g(z)$ defined by the above formula is an entire function given by

$$
g(z)=\sum_{n=0}^{\infty} \frac{(-1)^{n}}{(n+1) !} z^{n}=\frac{e^{-z}-1}{-z} \text { for } z \neq 0, g(0)=1,
$$


hence the function $g^{-1}(z)=\frac{1}{g(z)}$ is an analytic function in a neighborhood of 0 , more exactly for $z \neq 2 k \pi i, k= \pm 1, \pm 2, \ldots$, and we have the identities

$$
g^{-1}(z)=\frac{-z}{e^{-z}-1}, g^{-1}(-z)=g^{-1}(z)-z, g^{-1}(z)=1+\frac{1}{2} z+\ldots
$$

Defining $f(z)=g^{-1}(z)-\frac{1}{2} z$, we have $f(-z)=f(z)$, so

$$
f(z)=1+\sum_{p=1}^{\infty} k_{2 p^{2}} z^{2 p}, g^{-1}(z)=f(z)+\frac{1}{2} z, g^{-1}(-z)=f(z)-\frac{1}{2} z .
$$

Using (32) we can derive easily the following formulas:

$$
\frac{\partial Z(u, v)}{\partial u}=g^{-1}\left(-\operatorname{ad}_{Z(u, v)}\right) X, \frac{\partial Z(u, v)}{\partial v}=g^{-1}\left(\operatorname{ad}_{Z(u, v)}\right) Y .
$$

We apply them to derive the second-order Taylor expansion of $Z(u, v)$ with respect to the variable $u$, for example:

$$
\begin{aligned}
& Z(u, v)=Z(0, v)+u \frac{\partial Z(0, v)}{\partial u}+u^{2} \int_{0}^{1} d t(1-t) \frac{\partial^{2} Z(t u, v)}{\partial u^{2}} \\
& Z(0, v)=v Y, \frac{\partial Z(0, v)}{\partial u}=g^{-1}\left(-\operatorname{ad}_{v Y}\right) X=g^{-1}\left(-v \operatorname{ad}_{Y}\right) X
\end{aligned}
$$

From this we get

$$
\begin{gathered}
\frac{1}{i} \log e^{i X} e^{i Y}=Y+g^{-1}\left(-i \operatorname{ad}_{Y}\right) X+\mathscr{F}(X ; Y), \\
|\mathscr{F}(X ; Y)| \leqq O(1)|X|^{2}
\end{gathered}
$$

for $|X|,|Y|$ sufficiently small, where $O(1)$ is an absolute constant [e.g., we can take $O(1)=24$ for $\left.|X| \leqq \frac{1}{20},|Y| \leqq \frac{1}{12}\right]$. Another important function we will need later is

Repeating the above calculations, we get

$$
Z(u)=\log e^{u X+Y} e^{-Y} .
$$

$$
Z(u)=u g\left(-\operatorname{ad}_{Y}\right) X+u^{2} \int_{0}^{1} d t(1-t) Z^{\prime \prime}(t u)
$$

and

$$
\frac{1}{i} \log e^{i X+i Y} e^{-i Y}=g\left(-i \operatorname{ad}_{Y}\right) X+O\left(|X|^{2}\right) .
$$

\section{B. Compositions of Averaging Operations}

Let us recall the basic definitions. Let $U$ be a gauge field configuration with values in $\mathrm{U}(N)$. The one-step averaging operation is defined by

$$
\bar{U}_{c}=\exp \left[i \sum_{x \in B\left(c_{-}\right)} L^{-d} \frac{1}{i} \log U\left(\Gamma_{c, x}\right) U(c)^{-1}\right] U(c), c \subset \Omega^{(1)},
$$


and if $k^{\text {th }}$ order averaging $\bar{U}^{k}$ is defined at bonds of $\Omega^{(k)}$, then

$$
\bar{U}_{c}^{k+1}=\overline{\left(\bar{U}^{k}\right)_{c}}=\exp \left[i \sum_{x \in B(c-)} L^{-d} \frac{1}{i} \log \bar{U}^{k}\left(\Gamma_{c, x}\right)\left(\bar{U}^{k}(c)\right)^{-1}\right] \bar{U}^{k}(c), c \subset \Omega^{(k+1)},
$$

where the contours $\Gamma_{c, x}$ are defined on the lattice $\Omega^{(k)}$.

Let us notice that this definition is local in the sense that $\bar{U}_{c}^{k}, c \subset \Omega^{(k)}$, depends only on the bond variables $U_{b}$ for $b \subset B^{k}\left(c_{-}\right) \cup B^{k}\left(c_{+}\right)$. This property will play a very important role in the future. Let us notice also that the property (11) is satisfied. Indeed, for an arbitrary gauge field configuration $U$ and a gauge transformation $u$, we have $U^{u}\left(\Gamma_{c, x}\right) U^{u}(c)^{-1}=u\left(c_{-}\right) U\left(\Gamma_{c, x}\right) U(c)^{-1} u^{-1}\left(c_{-}\right)$; thus the matrices $U^{u}\left(\Gamma_{c, x}\right) U^{u}(c)^{-1}$ and $U\left(\Gamma_{c, x}\right) U(c)^{-1}$ are unitarily equivalent, their eigenvalues are equal, and by the definition (22) their logarithms are unitarily equivalent with the same unitary operator $u\left(c_{-}\right)$. Then from (42) we get $\left(\overline{U^{u}}\right)_{c}=u\left(c_{-}\right) \bar{U}_{c} u^{-1}\left(c_{+}\right)$.

Now we would like to understand how regular the configuration $\bar{U}^{k}$ is, assuming some regularity of $U$. We will investigate carefully the one-step averaging operation from this point of view. We assume that a configuration $V$ defined on a unit lattice $\Omega^{\prime}$ satisfies

$$
|V(\partial p)-1|<\alpha_{0}, \quad p \subset \Omega^{\prime},
$$

and $\alpha_{0}$ sufficiently small. We would like to get optimal bounds for $\left|\bar{V}\left(\partial p^{\prime}\right)-1\right|, p^{\prime}$ $C \Omega^{\prime(1)}$.

Let us denote by $y$ the upper right corner of the plaquette $p^{\prime}$ and let us introduce locally the axial gauge with the initial point $y$. This means that we take the contours

$$
\left.\Gamma_{y, x}=\left[y,\left(y_{1}, \ldots, y_{d-1}, x_{d}\right)\right] \cup \ldots \cup\left[y_{1}, x_{2}, \ldots, x_{d}\right), x\right]
$$

for $x$ in some neighborhood of $y$ containing $2^{d}$ blocks having the point $y$ as one of the corners, and we make a gauge transformation $v_{0}$ such that the gauge transformed configuration $V_{0}=V^{v_{0}}$ satisfies the conditions $V_{0}\left(\Gamma_{y, x}\right)=1$. Such a gauge transformation can be easily found because $V_{0}\left(\Gamma_{y, x}\right)=V^{v_{0}}\left(\Gamma_{y, x}\right)$ $=v_{0}(y) V\left(\Gamma_{y, x}\right) v_{0}^{-1}(x)=1$ implies $v_{0}(x)=v_{0}(y) V\left(\Gamma_{y, x}\right)$; thus $v_{0}$ is determined uniquely if $v_{0}(y)$ is given. Of course, we have

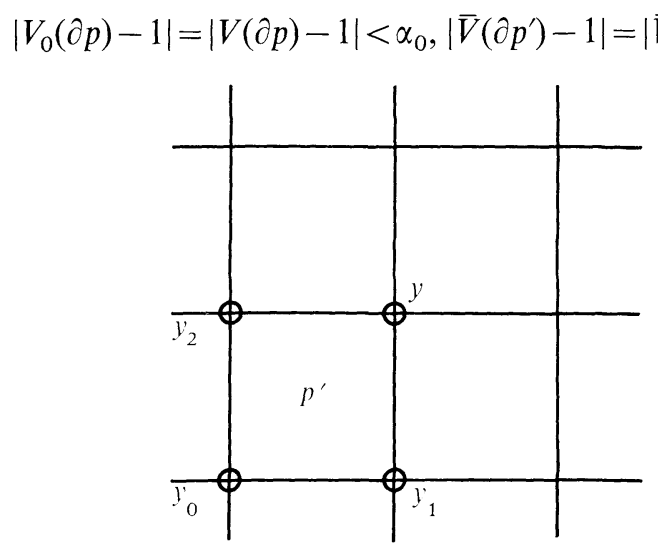

The conditions $V_{0}\left(\Gamma_{y, x}\right)=1$ imply $V_{0}\left(x, x+e_{1}\right)=1,\left|V_{0}\left(x, x+e_{2}\right)-1\right|<\left|x_{1}-y_{1}\right| \alpha_{0}$, $\left|V_{0}\left(x, x+e_{3}\right)-1\right|<\left(\left|x_{1}-y_{1}\right|+\left|x_{2}-y_{2}\right|\right) \alpha_{0}, \ldots, \quad\left|V_{0}\left(x, x+e_{\mu}\right)-1\right|<\left(\left|x_{1}-y_{1}\right|+\ldots\right.$ 
$\left.+\left|x_{\mu-1}-y_{\mu-1}\right|\right) \alpha_{0}, \mu=2, \ldots, d$, for $x$ in the neighborhood of $y$. If we denote

$$
\Delta\left(p^{\prime}\right)=B\left(y_{0}\right) \cup B\left(y_{1}\right) \cup B\left(y_{2}\right) \cup B(y),
$$

then for $b \subset \Delta\left(p^{\prime}\right)$ we have $\left|V_{0, b}-1\right|<\left|b_{-}-y\right| \alpha_{0} \leqq d L \alpha_{0}$, hence $V_{0, b}=e^{i A_{b}}$ and $\left|A_{b}\right|<2\left|b_{-}-y\right| \alpha_{0} \leqq 2 d L \alpha_{0}$. For $c \subset \partial p^{\prime}$, the contours $\Gamma_{c, x}$ are contained in $\Delta\left(p^{\prime}\right)$ and we have

$$
\begin{gathered}
\left|V_{0}\left(\Gamma_{c, x}\right)-1\right| \leqq \sum_{b \subset \Gamma_{c, x}}\left|V_{0, b}-1\right|<\left|\Gamma_{c, x}\right| d L \alpha_{0}<(2 d+1) L d L \alpha_{0}=O(1) L^{2} \alpha_{0}, \\
\left|V_{0}\left(\Gamma_{c, x}\right)-1-i A\left(\Gamma_{c, x}\right)\right| \leqq \frac{1}{2}\left(|A|\left(\Gamma_{c, x}\right)\right)^{2}<O(1)\left(L^{2} \alpha_{0}\right)^{2},
\end{gathered}
$$

so using the definition (21) of the logarithmic function, we have

$$
\left|\frac{1}{i} \log V_{0}\left(\Gamma_{c, x} \cup(-c)\right)-A\left(\Gamma_{c, x} \cup(-c)\right)\right|<O(1)\left(L^{2} \alpha_{0}\right)^{2}
$$

for $L^{2} \alpha_{0}$ sufficiently small. From this and (31) we get

$$
\begin{aligned}
\bar{V}_{0, c}= & \exp \left[i \sum_{x \in B\left(c_{-}\right)} L^{-d} A\left(\Gamma_{c, x} \cup(-c)\right)+O\left(\left(L^{2} \alpha_{0}\right)^{2}\right)\right] \\
& \cdot \exp \left[i A(c)+O\left(\left(L^{2} \alpha_{0}\right)^{2}\right)\right] \\
= & \exp \left[i \sum_{x \in B\left(c_{-}\right)} L^{-d} A\left(\Gamma_{c, x}\right)+O\left(\left(L^{2} \alpha_{0}\right)^{2}\right)\right],
\end{aligned}
$$

hence

$$
\left|\bar{V}_{0, c}-1-i \sum_{x \in B\left(c_{-}\right)} L^{-d} A\left(\Gamma_{c, x}\right)\right|<O(1)\left(L^{2} \alpha_{0}\right)^{2} .
$$

Now we can estimate $\left|\bar{V}_{0}\left(\partial p^{\prime}\right)-1\right|$. We have

$$
\left|\bar{V}_{0}\left(\partial p^{\prime}\right)-1-i \sum_{c \subset \partial p^{\prime}} \sum_{x \in B(c-)} L^{-d} A\left(\Gamma_{c, x}\right)\right|<O(1)\left(L^{2} \alpha_{0}\right)^{2} .
$$

Denoting by $\left(p^{\prime}\right)_{x}$ a plaquette obtained by translation of the plaquette $p^{\prime}$ to the point $x$, we have the identity

$$
\begin{aligned}
\sum_{c \subset \partial p^{\prime}} \sum_{x \in B\left(c_{-}\right)} L^{-d} A\left(\Gamma_{c, x}\right) & =\sum_{x \in B\left(y_{0}\right)} L^{-d} A\left(\partial\left(p^{\prime}\right)_{x}\right) \\
& =\sum_{x \in B\left(y_{0}\right)} L^{-d} \sum_{p \subset\left(p^{\prime}\right)_{x}} A(\partial p) .
\end{aligned}
$$

Further, we have for $p \subset \Delta\left(p^{\prime}\right)$

$$
\left|V_{0}(\partial p)-1-i A(\partial p)\right|<\frac{1}{2}(|A|(\partial p))^{2}<\frac{1}{2}\left(8 d L \alpha_{0}\right)^{2}=O(1) L^{2} \alpha_{0}^{2} .
$$

Gathering together the above three inequalities, we obtain

$$
\begin{aligned}
\left|\bar{V}_{0}\left(\partial p^{\prime}\right)-1\right| & <\sum_{x \in B\left(y_{0}\right)} L^{-d} \sum_{p \subset\left(p^{\prime}\right)_{x}}|A(\partial p)|+O(1)\left(L^{2} \alpha_{0}\right)^{2} \\
& <\sum_{x \in B\left(y_{0}\right)} L^{-d} \sum_{p \subset\left(p^{\prime}\right)_{x}}\left|V_{0}(\partial p)-1\right|+O(1)\left(L^{2} \alpha_{0}\right)^{2} \\
& <L^{2} \alpha_{0}+O(1)\left(L^{2} \alpha_{0}\right)^{2} .
\end{aligned}
$$

This is the estimate we are looking for. Let us notice that it is a local result; the bound above depends on bounds for $V(\partial p)-1$ on $\Delta\left(p^{\prime}\right)$, i.e., for $p \subset \Delta\left(p^{\prime}\right)$. We formulate the results in the following: 
Proposition 1. There exist positive constants $C_{0}, c_{2}^{\prime}$ such that for every configuration $V$ satisfying (44) for $p \subset \Delta\left(p^{\prime}\right)$ and for $\alpha_{0} \leqq c_{2}^{\prime}$, we have

$$
\left|\bar{V}\left(\partial p^{\prime}\right)-1\right|<L^{2} \alpha_{0}+C_{0}\left(L^{2} \alpha_{0}\right)^{2} .
$$

The constant $C_{0}$ depends on $d$ and $c_{2}^{\prime}$ depends on $d$ and $L$.

Now it becomes obvious what assumption we have to make for a configuration $U$ in order to get a bound on $\bar{U}^{k}(\partial p)-1, p \subset \Omega^{(k)}$. Each averaging operation rescales a bound on plaquette variables approximately by the factor $L^{2}$, hence $k$ operations by the factor $L^{2 k}$. To get some small number yet, we have to assume that

$$
|U(\partial p)-1|<\alpha_{0} \eta^{2}, \quad \eta=L^{-k}
$$

on some set of plaquettes. If $\alpha_{0} \leqq c_{2}^{\prime}$, then by the above Proposition, $\left|\bar{U}\left(\partial p^{\prime}\right)-1\right|$ $<L^{2} \alpha_{0} \eta^{2}+C_{0}\left(L^{2} \alpha_{0} \eta^{2}\right)$. We take $k \geqq 1$ so $\alpha_{0} L^{2} \eta^{2}+C_{0}\left(\alpha_{0} L^{2} \eta^{2}\right)^{2} \leqq \alpha_{0}+C_{0} \alpha_{0}^{2}$ and we assume further that $\alpha_{0}+C_{0} \alpha_{0}^{2} \leqq c_{2}^{\prime}$. Applying the Proposition again, we get for $p^{\prime \prime}$ $C \Omega^{(2)}$

$$
\begin{aligned}
\left|\bar{U}^{2}\left(\partial p^{\prime \prime}\right)-1\right|< & \alpha_{0} L^{4} \eta^{2}+C_{0} L^{2}\left(L^{2} \alpha_{0} \eta^{2}\right)^{2}+C_{0}\left(\alpha_{0} L^{4} \eta^{2}+C_{0} L^{2}\left(L^{2} \alpha_{0} \eta^{2}\right)^{2}\right)^{2} \\
= & \alpha_{0} L^{4} \eta^{2}+C_{0}\left(\alpha_{0} L^{4} \eta^{2}\right)^{2} \\
& +C_{0}\left(\alpha_{0} L^{4} \eta^{2}\right)^{2} L^{-2}\left[1+2 C_{0} \alpha_{0} L^{4} \eta^{2}+C_{0}^{2} L^{-2}\left(\alpha_{0} L^{4} \eta^{2}\right)^{2}\right] \\
\leqq & \alpha_{0} L^{4} \eta^{2}+C_{0}\left(\alpha_{0} L^{4} \eta^{2}\right)^{2}\left[1+L^{-2}\left(1+C_{0} \alpha_{0}\right)^{2}\right] .
\end{aligned}
$$

We make the following inductive assumption for $j \leqq k$ :

$$
\begin{aligned}
&\left|\bar{U}^{j}(\partial p)-1\right|<\alpha_{0} L^{2 j} \eta^{2}+C_{0}\left(\alpha_{0} L^{2 j} \eta^{2}\right)^{2} \\
& \cdot\left[1+L^{-2}\left(1+C_{0} \alpha_{0}\right)^{2}+\ldots+L^{-2(j-1)}\left(1+C_{0} \alpha_{0}\right)^{2(j-1)}\right] .
\end{aligned}
$$

The right-hand side can be bounded by $\alpha_{0}+C_{0} \alpha_{0}^{2} 2$ if $L^{-2}\left(1+C_{0} \alpha_{0}\right)^{2}<\frac{1}{2}$. The last inequality holds if, e.g., $C_{0} \alpha_{0} \leqq \frac{1}{3}$, and then $\alpha_{0}+C_{0} \alpha_{0}^{2} 2<2 \alpha_{0}$. We assume further that $2 \alpha_{0} \leqq c_{2}^{\prime}$. Then for $j<k$, we can apply Proposition 1 to the configuration $\bar{U}^{j}$ and we get for $p \subset \Omega^{(j+1)}$

$$
\begin{aligned}
\left|\bar{U}^{j+1}(\partial p)-1\right|< & \alpha_{0} L^{2(j+1)} \eta^{2}+C_{0} L^{2}\left(\alpha_{0} L^{2 j} \eta^{2}\right)^{2}[1+\ldots] \\
& +C_{0}\left(\alpha_{0} L^{2(j+1)} \eta^{2}+C_{0} L^{2}\left(\alpha_{0} L^{2 j} \eta^{2}\right)^{2}[1+\ldots]\right)^{2} \\
\leqq & \alpha_{0} L^{2(j+1)} \eta^{2}+C_{0}\left(\alpha_{0} L^{2(j+1)} \eta^{2}\right)^{2}\left\{1+[1+\ldots] L^{-2}\left(1+C_{0} \alpha_{0}\right)^{2}\right\} .
\end{aligned}
$$

Thus the inequality (53) is proved for all $j \leqq k$. Taking $j=k$, we get

Proposition 2. If $U$ satisfies (52) with $\alpha_{0} \leqq c_{2}=\min \left\{\frac{1}{3 C_{0}}, \frac{1}{2} c_{2}^{\prime}\right\}$, then

$$
\left|\bar{U}^{k}(\partial p)-1\right|<\alpha_{0}+2 C_{0} \alpha_{0}^{2}<2 \alpha_{0}, \quad p \subset \Omega^{(k)} .
$$

The result is local in the sense that if $p=\langle x, y, z, w\rangle$, then it is enough to assume (52) for $p \subset B^{k}(x) \cup B^{k}(y) \cup B^{k}(z) \cup B^{k}(w)$.

\section{Other Averaging Operations}

In future analysis of variational problems we will have a situation, where the averaging operation will be composed with a change of gauge conditions, i.e., with 
a gauge transformation. Let us recall that if we apply a gauge transformation $v$ to a configuration $V, V_{b}^{v}=v\left(b_{-}\right) V_{b} v^{-1}\left(b_{+}\right), b \subset \Omega^{\prime}$, then $\left(\overline{V^{v}}\right)_{c}=v\left(c_{-}\right) \bar{V}_{c} v^{-1}\left(c_{+}\right), c \subset \Omega^{(1)}$. We will consider gauge field configurations $V$ of the form $V=V^{\prime} V_{0}$, where $V_{0}$ is a fixed configuration and $V^{\prime}$ may have values in the complexified group $G^{c}$. We find easily that if we apply a gauge transformation $v$ to $V$ and we write $V^{v}=V^{v} V_{0}$, then

$$
V_{b}^{\prime v}=v\left(b_{-}\right) V_{b}^{\prime} R\left(V_{0, b}\right) v^{-1}\left(b_{+}\right)=v\left(b_{-}\right) V_{b}^{\prime} R_{0, b} v^{-1}\left(b_{+}\right),
$$

where for arbitrary invertible matrix $X$ the operator $R(X)$ is given by the formula

$$
R(X) Y=X Y X^{-1} \text {. }
$$

$R(X)$ acts on the algebra of all matrices and has the following properties:

$$
\begin{gathered}
R(X) f(Y)=f(R(X) Y) \text { for analytic functions } f, \\
R(X) R(Y)=R(X Y), \quad R(X)^{-1}=R\left(X^{-1}\right), \\
R(X)^{*}=R\left(X^{*}\right) .
\end{gathered}
$$

In a one-step renormalization transformation we consider configurations $V^{\prime}$ satisfying axial gauge conditions in blocks:

$$
\left(R_{0, y} V^{\prime}\right)\left(\Gamma_{y, x}\right)=\prod_{b \subset \Gamma_{y, x}} R\left(V_{0}\left(\Gamma_{y, b_{-}}\right)\right) V_{b}^{\prime}=1, x \in B(y), x \neq y .
$$

It will be convenient to change this gauge into another one. We apply a gauge transformation $v^{-1}$ to $V^{\prime}$ and we get a configuration $V_{1}$, thus

$$
V^{\prime}=V_{1}^{v} \quad \text { and } \quad \bar{V}_{c}=\left(\overline{V^{\prime} V_{0}}\right)_{c}=\left(\overline{V_{1}^{v} V_{0}}\right)_{c}=v(c)\left(\overline{V_{1} V_{0}}\right)_{c} v^{-1}\left(c_{+}\right) .
$$

We will consider this average for configurations $V^{\prime}$ and $V_{1}$ with values close to 1 and we will be interested in the expression

$$
\bar{V}_{c}\left(\overline{V_{0}}\right)_{c}^{-1}=v\left(c_{-}\right)\left(\overline{V_{1} V_{0}}\right)_{c}\left(\overline{V_{0}}\right)_{c}^{-1}{\overline{R_{0}}}_{c} v^{-1}\left(c_{+}\right), \overline{R_{0, c}}=R\left(\left(\overline{V_{0}}\right)_{c}\right) \text {. }
$$

The gauge conditions (58) written in terms of the configurations $V_{1}$ and $v$ have the form

$$
\begin{aligned}
\left(R_{0, y} V^{\prime}\right)\left(\Gamma_{y, x}\right) & =v(y)\left(R_{0, y} V_{1}\right)\left(\Gamma_{y, x}\right)\left(R_{0, y} v\right)^{-1}(x)=1, \\
\left(R_{0, y} v\right)(x) & =R\left(V_{0}\left(\Gamma_{y, x}\right)\right) v(x),
\end{aligned}
$$

and they imply

$$
\left(R_{0, y} v\right)(x)=v(y)\left(R_{0, y} V_{1}\right)\left(\Gamma_{y, x}\right), x \in B(y), x \neq y, y \in \Omega^{(1)} .
$$

To determine the configuration $v$ uniquely, we will impose on it an additional condition at each block.

To find a form of this condition, let us recall the simplest case of one vector field considered in paper [1]. To change a gauge, we make a gauge transformation $\lambda$ satisfying $\left(Q^{\prime} \lambda\right)(y)=\sum_{x \in B(y)} L^{-d} \lambda(x)=0$. Such a transformation does not change the average given by (1.11) in [2], and this is very important because the explicit representations of propagators, and other formulas, hold for this specific form of the averaging operation. In the considered general case, we will also try to find the additional condition on gauge transformation requiring that the form of the 
averaging operation should be preserved. We will be able to obtain only an approximate invariance. Because of the axial gauge conditions (58), the average $\left(\overline{V^{\prime} V_{0}}\right)_{c}$ depends on $\left(R_{0, c_{-}} V^{\prime}\right)([x, x(c)])=\prod_{b \subset[x, x(c)]} R\left(V_{0}\left(\Gamma_{c_{-}, x} \cup\left[x, b_{-}\right]\right)\right) V_{b}^{\prime}$, $x \in B\left(c_{-}\right)$, and a good approximation of the function $\frac{1}{i} \log \left(\overline{V^{\prime} V_{0}}\right)_{c}\left(\bar{V}_{0}^{-}\right)_{c}^{-1}$ for $V^{\prime}=e^{i A}, A$ small, is given by $\left(Q_{0} A\right)(c)=\sum_{x \in B\left(c_{-}\right)} L^{-d}\left(R_{0, c_{-}} A\right)([x, x(c)])$, where $R_{0, c_{-}} A$ is defined as $R_{0, c_{-}} V^{\prime}$, only the product over $b$ is replaced by the sum. If we make a small gauge transformation $v=e^{i \lambda}$, then a good approximation of this transformation acting on Lie algebra variables $A$ is given by $A_{b}^{\lambda}=A_{b}-\left(R_{0, b} \lambda\left(b_{+}\right)\right.$ $\left.-\lambda\left(b_{-}\right)\right)=A_{b}-\left(D_{V_{0}} \lambda\right)(b)$. Under such a transformation the average $Q_{0} A$ changes as follows:

$$
\begin{aligned}
\left(Q_{0} A^{\lambda}\right)(c)= & \sum_{x \in B\left(c_{-}\right)} L^{-d}\left(R_{0, c_{-}} \lambda\right)(x)+\left(Q_{0} A\right)(c) \\
& -\sum_{x^{\prime} \in B\left(c_{+}\right)} L^{-d}\left(R_{0, c_{-}} \lambda\right)\left(x^{\prime}\right) .
\end{aligned}
$$

If we define $\left(Q_{0}^{\prime} \lambda\right)(y)=\sum_{x \in B(y)} L^{-d}\left(R_{0, y} \lambda\right)(x)$, then the first term in the right-hand side above is equal to $\left(Q_{0}^{\prime} \lambda\right)\left(c_{-}\right)$. There are troubles with the second term because

$$
\begin{array}{r}
\sum_{x^{\prime} \in B\left(c_{+}\right)} L^{-d}\left(R_{0, c_{-}} \lambda\right)\left(x^{\prime}\right)=\sum_{x^{\prime} \in B\left(c_{+}\right)} L^{-d} R\left(V_{0}\left(\Gamma_{c_{-}, x} \cup\left[x, x^{\prime}\right]\right)\right) \lambda\left(x^{\prime}\right) \\
=\sum_{x^{\prime} \in B\left(c_{+}\right)} L^{-d} R\left(V_{0}\left(\Gamma_{c, x} \cup(-c)\right)\right) R\left(V_{0}(c)\right)\left(R_{0, c_{+}} \lambda\right)\left(x^{\prime}\right),
\end{array}
$$

and this expression is only approximately equal to $R\left(V_{0}(c)\right)\left(Q_{0}^{\prime} \lambda\right)\left(c_{+}\right)$, because $V_{0}\left(\Gamma_{c, x} \cup(-c)\right)$ are close to 1 for $V_{0}$ regular, but not necessarily equal to 1 . Now if we assume that $\lambda$ satisfies the conditions $Q_{0}^{\prime} \lambda=0$, then the form of $Q_{0} A$ is approximately preserved under such a gauge transformation. Finally, these conditions are approximations for $\lambda$ small to the conditions

$$
\left(\overline{R_{0} v}\right)(y)=v(y) \exp \left[i \sum_{x \in B(y)} L^{-d} \frac{1}{i} \log v^{-1}(y)\left(R_{0, y} v\right)(x)\right]=1 .
$$

We assume that the gauge transformation $v$ we have applied to the configuration $V^{\prime}$ satisfies these conditions. The equality (60) and the condition (61) imply

$$
v^{-1}(y)\left(R_{0, y} v\right)(x)=\left(R_{0, y} V_{1}\right)\left(\Gamma_{y, x}\right)
$$

and

$$
v(y)=\exp \left[-i \sum_{x \in B(y)} L^{-d} \frac{1}{i} \log \left(R_{0, y} V_{1}\right)\left(\Gamma_{y, x}\right)\right] .
$$

Thus the gauge transformation $v$ is determined uniquely by the gauge conditions (58) and conditions (61). It is a function of the gauge transformed configuration $V_{1}$. From (59) and (62) we get

$$
\begin{aligned}
\bar{V}_{c}\left(\overline{V_{0}}\right)_{c}^{-1}= & \exp \left[-i \sum_{x \in B\left(c_{-}\right)} L^{-d} \frac{1}{i} \log \left(R_{0, c_{-}} V_{1}\right)\left(\Gamma_{c_{-}, x}\right)\right]\left(\overline{V_{1} V_{0}}\right)_{c}\left(\overline{V_{0}}\right)_{c}^{-1} \\
& \cdot \overline{R_{0, c}} \exp \left[i \sum_{x^{\prime} \in B(c+)} L^{-d} \frac{1}{i} \log \left(R_{0, c_{+}} V_{1}\right)\left(\Gamma_{c_{+}, x^{\prime}}\right)\right]
\end{aligned}
$$


The same reasoning can be applied to a higher order average $\bar{U}^{k}$, where $U=U^{\prime} U_{0}, U_{0}$ is a fixed configuration and $U^{\prime}$ satisfies a sequence of axial gauge conditions in blocks of lattices $\Omega, \Omega^{(1)}, \ldots, \Omega^{(k-1)}$. More precisely, we describe these conditions in the following way. For blocks of the lattice $\Omega$, we assume

$$
\left(R_{0, y} U^{\prime}\right)\left(\Gamma_{y, x}\right)=1, x \in B(y), x \neq y, y \in \Omega^{(1)},
$$

where $R_{0}$ is defined by the configuration $U_{0}$. Next defining

$$
\left(\tilde{U^{\prime}}\right)_{c}=\left(\overline{U^{\prime} U_{0}}\right)_{c}\left(\overline{U_{0}}\right)_{c}^{-1}
$$

we assume

$$
\left(\overline{R_{0, z}} \tilde{U}^{\prime}\right)\left(\Gamma_{z, y}\right)=1, y \in B(z), y \neq z, z \in \Omega^{(2)},
$$

and $\overline{R_{0}}$ is defined by $\overline{U_{0}}$. If an average $\tilde{U}^{\prime j}$ is defined on the lattice $\Omega^{(j)}, j<k$, then we assume

$$
\left(\bar{R}_{0, y}^{j} \tilde{U}^{\prime j}\right)\left(\Gamma_{y, x}\right)=1, x \in B(y), x \neq y, y \in \Omega^{(j+1)},
$$

and we define

$$
\tilde{U}_{c}^{j+1}=\left(\overline{\widetilde{U}^{\prime j} \bar{U}_{0}^{j}}\right)_{c}\left(\overline{\bar{U}_{0}^{j}}\right)_{c}^{-1} .
$$

From this inductive definition of the average $\tilde{U}^{\prime j}$, it follows easily that

$$
\tilde{U}_{b}^{\prime j}=\left(\overline{U^{\prime} U_{0}}\right)_{b}^{j}\left(\bar{U}_{0}^{j}\right)_{b}^{-1}, \quad b \subset \Omega^{(j)} .
$$

Now we would like to change these gauge conditions and we apply a gauge transformation $u^{-1}$ to $U^{\prime}$. We get some configuration $U_{1}$ and $U^{\prime}=U_{1}^{u}$. The $j^{\text {th }}$ order averages $\bar{U}^{j}$ transform as follows,

$$
\overline{U_{b}^{j}}=\left(\overline{U_{1}^{u} U_{0}}\right)_{b}^{j}=u\left(b_{-}\right)\left(\overline{U_{1} U_{0}}\right)_{b}^{j} u^{-1}\left(b_{+}\right), \quad b \subset \Omega^{(j)},
$$

and this implies a transformation law for $\tilde{U}^{j}$,

$$
\tilde{U}_{b}^{\prime j}=\left(\tilde{U}_{1}^{u}\right)_{b}^{j}=u\left(b_{-}\right)\left(\tilde{U}_{1}\right)_{b}^{j} \bar{R}_{0, b}^{j} u^{-1}\left(b_{+}\right), \quad b \subset \Omega^{(j)} .
$$

The gauge conditions (67) written in terms of $U_{1}$ give us the following equations:

$$
\left(\bar{R}_{0, y}^{j} \tilde{U}^{j}\right)\left(\Gamma_{y, x}\right)=u(y)\left(\bar{R}_{0, y}^{j} \tilde{U}_{1}^{j}\right)\left(\Gamma_{y, x}\right)\left(\bar{R}_{0, y}^{j} u\right)^{-1}(x)=1
$$

for $x \in B(y), x \neq y, y \in \Omega^{(j+1)}, j<k$. Solving these equations, we get

$$
\begin{gathered}
\left(R_{0, x_{1}} u\right)(x)=u\left(x_{1}\right)\left(R_{0, x_{1}} U_{1}\right)\left(\Gamma_{x_{1}, x}\right) \text { for } \quad x \in B\left(x_{1}\right), x_{1} \in \Omega^{(1)}, \\
\left(\overline{R_{0, x_{2}}} u\right)\left(x_{1}\right)=u\left(x_{2}\right)\left(\overline{R_{0, x_{2}}} \tilde{U}_{1}\right)\left(\Gamma_{x_{2}, x_{1}}\right) \text { for } \quad x_{1} \in B\left(x_{2}\right), x_{2} \in \Omega^{(2)},
\end{gathered}
$$

hence

$\left(\overline{R_{0, x_{2}}} R_{0, x_{1}} u\right)(x)=u\left(x_{2}\right)\left(\overline{R_{0, x_{2}}} \tilde{U}_{1}\right)\left(\Gamma_{x_{2}, x_{1}}\right) \overline{R_{0, x_{2}}}\left(R_{0, x_{1}} U_{1}\right)\left(\Gamma_{x_{1}, x}\right), x \in B^{2}\left(x_{2}\right)$,

and for arbitrary $j<k$ we have

$\left(\bar{R}_{0, x_{j+1}}^{j} u\right)\left(x_{j}\right)=u\left(x_{j+1}\right)\left(\bar{R}_{0, x_{j+1}}^{j} \tilde{U}_{1}^{j}\right)\left(\Gamma_{x_{j+1}, x_{j}}\right)$ for $x_{j} \in B\left(x_{j+1}\right), x_{j+1} \in \Omega^{(j+1)}$.

Let us denote $U_{0}\left(\Gamma_{x_{j}, x}^{(j)}\right)=\bar{U}_{0}^{j-1}\left(\Gamma_{x_{j}, x_{j-1}}\right) \cdot \ldots \cdot \bar{U}_{0}\left(\Gamma_{x_{2}, x_{1}}\right) U_{0}\left(\Gamma_{x_{1}, x}\right)$, then $\left(\bar{R}_{0, x_{j+1}}^{j} \bar{R}_{0, x_{j}}^{j-1} \cdot \ldots \cdot \bar{R}_{0, x_{2}} R_{0, x_{1}} u\right)(x)=R\left(U_{0}\left(\Gamma_{x_{j+1}, x}^{(j+1)}\right)\right) u(x)$, and we have the equality 


$$
\begin{aligned}
R\left(U_{0}\left(\Gamma_{x_{j+1}, x}^{(j+1)}\right)\right) u(x)= & u\left(x_{j+1}\right)\left(\bar{R}_{0, x_{j+1}}^{j} \tilde{U}_{1}^{j}\right)\left(\Gamma_{x_{j+1}, x_{j}}\right) \bar{R}_{0, x_{j+1}}^{j} \\
& \cdot\left(\bar{R}_{0, x_{j}}^{j-1} \tilde{U}_{1}^{j-1}\right)\left(\Gamma_{x_{j}, x_{j-1}}\right) \cdot \ldots \cdot \bar{R}_{0, x_{j+1}}^{j} \cdot \ldots \cdot \bar{R}_{0, x_{2}} \\
& \cdot\left(R_{0, x_{1}} U_{1}\right)\left(\Gamma_{x_{1}, x}\right)=u\left(x_{j+1}\right)\left(\bar{R}_{0, x_{j+1}}^{j} \tilde{U}_{1}^{j}\right)\left(\Gamma_{x_{j+1}, x_{j}}\right) \\
& \cdot R\left(\bar{U}_{0}^{j}\left(\Gamma_{x_{j+1}, x_{j}}^{(1)}\right)\left(\bar{R}_{0, x_{j}}^{j-1} \tilde{U}_{1}^{j-1}\right)\left(\Gamma_{x_{j}, x_{j-1}}\right) \cdot \ldots\right. \\
& \cdot R\left(\bar{U}_{0}\left(\Gamma_{x_{j+1}, x_{1}}^{(j)}\right)\left(R_{0, x_{1}} U_{1}\right)\left(\Gamma_{x_{1}, x}\right)\right. \\
= & u\left(x_{j+1}\right)\left(R_{0, x_{j+1}} U_{1}\right)\left(\Gamma_{x_{j+1}, x}^{(j+1)}\right), x \in B^{j+1}\left(x_{j+1}\right)
\end{aligned}
$$

where the symbol $\left(R_{0, x_{j+1}} U_{1}\right)\left(\Gamma_{x_{j+1}, x}^{(j+1)}\right)$ is defined by the last equation. Taking (77) for $j=k-1$, we may determine the gauge transformation $u$ uniquely, given values $u(y)$ at points $y$ of the lattice $\Omega^{(k)}$. We calculate these values from additional conditions, as in (61) and (62). These conditions are straightforward generalizations of the conditions (61). At first we define inductively a $k^{\text {th }}$ order averaging operation for gauge transformations. Generally a one-step averaging transformation defined by a field configuration $V_{0}$ is given by

$$
\begin{aligned}
\left(\overline{R_{0} v}\right)(y) & =\left(\overline{R\left(V_{0}\right) v}\right)(y)=\left\{\left(\overline{\left.R\left(V_{0}\right) v\right)(x)}\right\}_{x \in B(y)}\right. \\
& =v(y) \exp \left[i \sum_{x \in B(y)} L^{-d} \frac{1}{i} \log v^{-1}(y) R\left(V_{0}\left(\Gamma_{y, x}\right)\right) v(x)\right],
\end{aligned}
$$

where $v$ is defined on a lattice $\Omega^{\prime}, y \in \Omega^{\prime(1)}$.

For a given configuration $U_{0}$ we define inductively

$$
\begin{gathered}
\left(\overline{R_{0} u}\right)\left(x_{1}\right)=\left(\overline{R\left(U_{0}\right) u}\right)\left(x_{1}\right), \quad x_{1} \in \Omega^{(1)}, \\
\left(\overline{R_{0} u^{j+1}}\right)\left(x_{j+1}\right)=\left(\overline{R\left(\bar{U}_{0}^{j}\right) \bar{R}_{0} u^{j}}\right)\left(x_{j+1}\right), \quad x_{j+1} \in \Omega^{(j+1)} .
\end{gathered}
$$

The additional conditions are

$$
\left(\overline{R_{0}} u^{k}\right)(y)=1, \quad y \in \Omega^{(k)} .
$$

Now using the identities (76), (77) we will solve the equations (81) and we will determine $u$ uniquely as a function of $U_{1}$. We have

$$
\left(\overline{R_{0} u}\right)\left(x_{1}\right)=u\left(x_{1}\right)\left(\overline{\left.R_{0, x_{1}} U_{1}\right)\left(\Gamma_{x_{1}, .}\right.}\right)=u\left(x_{1}\right) \overline{R_{0, x_{1}} U_{1}}
$$

for $x_{1} \in \Omega^{(1)}$, where the expression ${\overline{R_{0, x_{1}}}}_{1}$ is defined by the last equation. Next from (74) we have

$$
\begin{aligned}
& \left(\overline{R_{0} u^{2}}\right)\left(x_{2}\right)=\overline{\left(R\left(\bar{U}_{0}\right) \overline{R_{0} u}\right)}\left(x_{2}\right) \\
& \left.=\overline{\left\{\left(\overline{R_{0, x_{2}}} u\right)\left(x_{1}\right)\left(\overline{R_{0, x_{2}}} \overline{R_{0, x_{1}} U_{1}}\right)\left(x_{1}\right)\right.}\right\}_{x_{1} \in B\left(x_{2}\right)}
\end{aligned}
$$

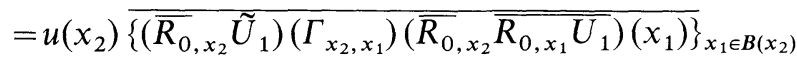

$$
\begin{aligned}
& =u\left(x_{2}\right) \overline{R_{0, x_{2}} U_{1}}{ }^{(2)}, \quad x_{2} \in \Omega^{(2)},
\end{aligned}
$$

where the last equation defines the symbol $\overline{R_{0, x_{2}} U_{1}}{ }^{(2)}$. We easily find by induction that

$$
\left({\overline{R_{0}}}^{j}\right)\left(x_{j}\right)=u\left(x_{j}\right){\overline{R_{0, x_{j}} U_{1}}}^{(j)}, \quad x_{j} \in \Omega^{(j)},
$$

where $\overline{R_{0, x_{j}} U_{1}}{ }^{(j)}$ is defined inductively as 


$$
\begin{aligned}
& \overline{R_{0, x_{j+1}} U_{1}}(j+1)
\end{aligned}
$$

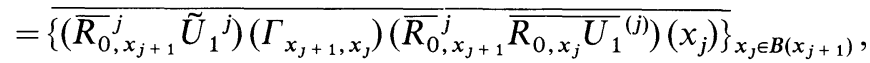

$$
\begin{aligned}
& x_{j+1} \in \Omega^{(j+1)} \text {. }
\end{aligned}
$$

The formula (84) together with the conditions (81) give the equation

$$
\left(\overline{R_{0} u^{k}}\right)(y)=u(y){\overline{R_{0, y} U_{1}}}^{(k)}=1 \text { for } y \in \Omega^{(k)},
$$

hence

$$
u(y)=\left(\overline{R_{0, y} U_{1}}{ }^{(k)}\right)^{-1} .
$$

Thus the gauge transformation is uniquely determined by all the conditions and is given by the formulas (77) for $j=k-1$ and by (87). Similarly as in (63) we get

$$
\begin{aligned}
\bar{U}_{b}^{k}\left(\bar{U}_{0}^{k}\right)_{b}^{-1} & =\left({\overline{U^{\prime} U_{0}}}^{k}\right)_{b}\left(\bar{U}_{0}^{k}\right)_{b}^{-1}=u\left(b_{-}\right)\left(\overline{U_{1} U_{0}}{ }^{k}\right)_{b}\left(\bar{U}_{0}^{k}\right)_{b}^{-1} \bar{R}_{0, b}^{k} u^{-1}\left(b_{+}\right) \\
& =\left(\overline{R_{0, b_{-}} U_{1}}{ }^{(k)}\right)^{-1} \tilde{U}_{1}^{k} \bar{R}_{0, b}^{k} \overline{R_{0, b_{+}} U_{1}}{ }^{(k)}, \quad b \subset \Omega^{(k)} .
\end{aligned}
$$

We may consider this expression as a new averaging operation of $k^{\text {th }}$ order acting on a configuration $U_{1}$ defined at bonds of the lattice $\Omega$. A result of the averaging is a configuration defined at bonds of the lattice $\Omega^{(k)}$. Such an operation for $k=1$ is given by the formula (63).

Now we will prove the following fundamental fact: the new $k^{\text {th }}$ order averaging operation defined by the last expression in (88) is a composition of $k$ averaging operations defined by (63) with properly chosen configurations $V_{0}$. More precisely, for a $j^{\text {th }}$ factor in this composition, we take $V_{0}=\bar{U}_{0}^{j-1}$. Let us introduce some new notations. We denote the averaging operation in (63) by

$$
\begin{aligned}
\left(\overline{\overline{R\left(V_{0}\right) V_{1}}}\right)_{c} & =\left(\overline{R_{0, c_{-}} V_{1}}\right)^{-1}\left(\overline{V_{1} V_{0}}\right)_{c}\left(\bar{V}_{0}\right)_{c}^{-1} \bar{R}_{0, c} \overline{R_{0, c_{+}} V_{1}} \\
& =\left(\overline{R_{0, c_{-}} V_{1}}\right)^{-1} \tilde{V}_{1} \bar{R}_{0, c} \overline{R_{0, c_{+}} V_{1}} .
\end{aligned}
$$

We define inductively a sequence of averaging operations of $j^{\text {th }}$ order composing $j$ operations defined above for $V_{0}=U_{0}, \bar{U}_{0}, \ldots, \bar{U}_{0}^{j-1}$ correspondingly. For $j=1$ we define

$$
\bar{U}_{1}=\overline{\overline{R\left(U_{0}\right) U_{1}}},
$$

and if the operation $\bar{U}_{1}^{j}$ is defined, then

$$
\overline{\bar{U}}_{1}^{j+1}=\overline{\overline{R\left(\bar{U}_{0}^{j}\right) \overline{\bar{U}}_{1}^{j}}}
$$

i.e., it is a composition of the operation (89) for $V_{0}=\bar{U}_{0}^{j}$ and of the $j^{\text {th }}$ order operation $\overline{\bar{U}}_{1}^{j}$.

Let us remark that these averages have the same locality properties as the averages $\bar{U}^{k}$, namely $\left(\bar{U}^{k}\right)_{c}, c \subset \Omega^{(k)}$, depends on the variables $U_{b}$ for $b \subset B^{k}\left(c_{-}\right)$ $\cup B^{k}\left(c_{+}\right)$.

We are going to prove now the fundamental equality

$$
\left(\overline{R_{0, b_{-} U_{1}}{ }^{(k)}}\right)^{-1} \tilde{U}_{1}^{k} \bar{R}_{0, b}^{k}{\overline{R_{0, b_{+}} U_{1}}}^{(k)}=\left(\bar{U}_{1}^{k}\right)_{b} .
$$

The proof will be by induction. For $k=1$ the equality holds by the definitions (89), (90), and the Eq. (63). If we have a configuration $V=V_{1} V_{0}$ on a lattice $\Omega^{\prime}$, then 
for an arbitrary gauge transformation $v$ we write $V=\left(V^{v^{-1}}\right)^{v}=\left(V_{1}^{v^{-1}} V_{0}\right)^{v}$ and we have

$$
\left(\tilde{V}_{1}\right)_{c}=\left(\overline{V_{1} V_{0}}\right)_{c} \bar{V}_{0,-c}=v\left(c_{-}\right)\left(\widetilde{V_{1}^{v^{-1}}}\right)_{c} \bar{R}_{0, c} v^{-1}\left(c_{+}\right), \quad c \subset \Omega^{\prime(1)} .
$$

Let us consider the case $k=2$, and let us start with an analysis of $\tilde{U}_{1}^{2}$. We have by (68) $\tilde{U}_{1}^{2}=\left(\widetilde{U}_{1}\right)$ and we apply the above identity with $V_{1}=\widetilde{U}_{1}, V_{0}=\bar{U}_{0}$, and $v(x)=\overline{R_{0, x} U_{1}}, x \in \Omega^{(1)}$ :

$$
\left(\tilde{U}_{1}^{2}\right)_{c}=\overline{R_{0, c_{-}} U_{1}}\left(\overline{\tilde{U}_{1}^{v^{-1}}}\right)_{c} \bar{R}_{0, c}^{2}\left(\overline{R_{0, c_{+}} U_{1}}\right)^{-1}, \quad c \subset \Omega^{(2)}
$$

Further, by the definition (89), we have

$$
\left(\tilde{U}_{1}^{v^{-1}}\right)_{b}=\left(\overline{R_{0, b_{-}} U_{1}}\right)^{-1} \tilde{U}_{1, b} \bar{R}_{0, b} \overline{R_{0, b_{+}} U_{1}}=\bar{U}_{1},
$$

hence

$$
\begin{aligned}
\left(\tilde{U}_{1}^{2}\right)_{c}= & \overline{R_{0, c_{-}} U_{1}}\left(\tilde{\bar{U}}_{1}\right)_{c} \bar{R}_{0, c}^{2}\left(\overline{R_{0, c+} U_{1}}\right)^{-1}=\overline{R_{0, c_{-}} U_{1}}\left(\overline{\bar{R}_{0, c-} \overline{\bar{U}}_{1}}\right) \\
& \cdot\left[\left(\overline{\bar{R}}_{0, c_{-}} \overline{\bar{U}}_{1}\right)^{-1}\left(\tilde{\overline{\bar{U}}}_{1}\right)_{c} \bar{R}_{0, c}^{2}\left(\overline{\bar{R}}_{0, c_{+}} \overline{\bar{U}}_{1}\right)\right] \bar{R}_{0, c}^{2}\left({\overline{\overline{R_{0, c+}}}}_{0, \bar{U}_{1}}\right)^{-1} \\
& \cdot \bar{R}_{0, c}^{2}\left(\overline{R_{0, c_{+}} U_{1}}\right)^{-1} .
\end{aligned}
$$

The expression in the square bracket above is equal to $\bar{U}_{1}^{2}$. Let us make the following inductive hypothesis:

$$
\begin{aligned}
\left(\tilde{U}_{1}^{j}\right)_{b} & =v_{j}\left(b_{-}\right)\left(\overline{\bar{U}}_{1}^{j}\right)_{b} \bar{R}_{0, b}^{j} v_{j}^{-1}\left(b_{+}\right)=\left(\overline{\bar{U}}_{1}^{j}\right)_{b}^{v_{j}}, \quad b \subset \Omega^{(j)}, \\
v_{j}(x) & \left.=\left(\bar{R}_{0, x} U_{1}\right) \overline{\left(\bar{R}_{0, x} \overline{\bar{U}}_{1}\right.}\right) \cdot \ldots \cdot\left(\overline{\bar{R}}_{0, x}^{j-1} \overline{\bar{U}}_{1}^{j-1}\right), \quad x \in \Omega^{(j)} .
\end{aligned}
$$

We have proved it for $j=1,2$. The definition (68) and the hypothesis imply

$$
\begin{aligned}
\left(\tilde{U}_{1}^{j+1}\right)_{c}= & \left(\left(\overline{\left.\bar{U}_{1}^{j}\right)^{v_{j}} \bar{U}_{0}^{j}}\right)_{c} \bar{U}_{0,-c}^{j+1}=v_{j}\left(c_{-}\right)\left(\tilde{\bar{U}}_{1}^{j}\right)_{c} \bar{R}_{0, c}^{j+1} v_{j}^{-1}\left(c_{+}\right)\right. \\
= & v_{j}\left(c_{-}\right)\left(\overline{\bar{R}}_{0, c_{-}}^{j} \overline{\bar{U}}_{1}^{j}\right)\left[\left(\overline{R_{0, c_{-}}^{j} \overline{\bar{U}}_{1}^{j}}\right)^{-1}\left(\tilde{\bar{U}}_{1}^{j}\right)_{c} \bar{R}_{0, c}^{j+1}\left(\overline{\bar{R}_{0, c_{+}}^{j} \overline{\bar{U}}_{1}^{j}}\right)\right] \\
& \cdot \bar{R}_{0, c}^{j+1}\left(\overline{\bar{R}_{0, c_{+}}^{j} \overline{\bar{U}}_{1}^{j}}\right)^{-1} \bar{R}_{0, c}^{j+1} v_{j}^{-1}\left(c_{+}\right) \\
= & v_{j+1}\left(c_{-}\right)\left(\overline{\bar{U}}_{1}^{j+1}\right)_{c} R_{0, c}^{j+1} v_{j+1}^{-1}\left(c_{+}\right),
\end{aligned}
$$

where we have used the identity (93) again and the other definitions.

Let us now consider the expressions $\overline{R_{0, x} U_{1}}{ }^{(j)}, x \in \Omega^{(j)}$. We will prove that

$$
\overline{R_{0, x} U_{1}}{ }^{(j)}=v_{j}(x), \quad x \in \Omega^{(j)} .
$$

For $j=1$ it is the definition (97) of $v_{j}$. For $j+1$ we have by (85), (97), and (99)

$$
\begin{aligned}
& {\overline{R_{0, y} U_{1}}}^{(j+1)}={\overline{\left\{\left(\bar{R}_{0, y}^{j} \tilde{U}_{1}^{j}\right)\left(\Gamma_{y, x}\right)\left(\bar{R}_{0, y}^{j}{\overline{R_{0, x} U_{1}}}^{(j)}\right)(x)\right.}}_{x \in B(y)} \\
& ={\overline{\left\{\left(\bar{R}_{0, y}^{j}\left(\bar{U}_{1}^{j}\right)^{v_{j}}\right)\left(\Gamma_{y, x}\right)\left(\bar{R}_{0, y}^{j} v_{j}\right)(x)\right\}_{x \in B(y)}}} \\
& =v_{j}(y)\left(\overline{\bar{R}_{0, y}^{j} \overline{\bar{U}}_{1}^{j}}\right)=v_{j+1}(y), \quad y \in \Omega^{(j+1)},
\end{aligned}
$$

hence the identity (99) is proved by induction. From (97), (99) for $j=k$ we get (92).

Let us consider again the gauge transformation $u$ calculated before in terms of $U_{1}$ and given by the equalities (77) for $j=k-1,(87)$. In the future we will need this transformation expressed by the averagings $\overline{\bar{U}}_{1}^{j}$. Let us write explicitly the formula 
for $u$

$$
\begin{aligned}
R\left(U_{0}\left(\Gamma_{y, x}^{(k)}\right)\right) u(x)= & \left(\overline{R_{0, y} U_{1}}{ }^{(k)}\right)^{-1} \prod_{j=k-1}^{0} R\left(\bar{U}_{0}^{j+1}\left(\Gamma_{y, x_{j}+1}^{(k-j-1)}\right)\right) \\
& \cdot\left(\bar{R}_{0, x_{j+1}}^{j} \tilde{U}_{1}^{j}\right)\left(\Gamma_{x_{j+1}, x_{j}}\right),
\end{aligned}
$$

where $x \in B^{k}(y), y \in \Omega^{(k)}, x_{k}=y, x_{0}=x$.

We use the equalities (97) and we get for $j>0$

$$
\begin{aligned}
\left(\bar{R}_{0, x_{j}+1}^{j} \tilde{U}_{1}^{j}\right)\left(\Gamma_{x_{j+1}, x_{j}}\right)= & v_{j}\left(x_{j+1}\right)\left(\bar{R}_{0, x_{j+1}}^{j} \bar{U}_{1}^{j}\right)\left(\Gamma_{x_{j+1}, x_{j}}\right) \\
& \cdot\left(\bar{R}_{0, x_{j+1}}^{j} v_{j}\right)^{-1}\left(x_{j}\right) .
\end{aligned}
$$

If we take two neighboring factors for $j, j-1$ in (101), then the last factor on the right-hand side above for $j$ and the first factor for $j-1$ give the product

$$
\begin{aligned}
& \left(R\left(\bar{U}_{0}^{j+1}\left(\Gamma_{y, x_{j+1}}^{(k-j-1)}\right)\right) R\left(\bar{U}_{0}^{j}\left(\Gamma_{x_{j+1}, x_{j}}\right)\right) v_{j}^{-1}\left(x_{j}\right)\right)\left(R\left(\bar{U}_{0}^{j}\left(\Gamma_{y, x_{j}}^{(k-j)}\right)\right) v_{j-1}\left(x_{j}\right)\right) \\
& =R\left(\bar{U}_{0}^{j}\left(\Gamma_{y, x_{j}}^{(k-j)}\right)\right)\left(v_{j}^{-1}\left(x_{j}\right) v_{j-1}\left(x_{j}\right)\right)=R\left(\bar{U}_{0}^{j}\left(\Gamma_{y, x_{j}}^{(k-j)}\right)\right)\left(\overline{\bar{R}}_{0, x_{j}}^{j-1} \bar{U}_{1}^{j-1}\right)^{-1},
\end{aligned}
$$

where we have used the second Eq. (97). We connect this expression with the factor corresponding to $j-1$. We get such an expression for $j-1=0$ also. The first factor in (102) for $j+1=k$ and the first factor on the right-hand side of (101) give $v_{k}^{-1}(y) v_{k-1}(y)$ and this is equal to (103) for $j=k$. Thus we obtain the equality

$$
\begin{aligned}
R\left(U_{0}\left(\Gamma_{y, x}^{(k)}\right)\right) u(x)= & \prod_{j=k-1}^{0} R\left(\bar{U}_{0}^{j+1}\left(\Gamma_{y, x_{j}+1}^{(k-j-1)}\right)\right) \\
& \cdot\left[\left(\overline{\bar{R}_{0, x_{j}+1}^{j} \bar{U}_{1}^{j}}\right)^{-1}\left(\bar{R}_{0, x_{j+1}}^{j} \overline{\bar{U}}_{1}^{j}\right)\left(\Gamma_{x_{j+1}, x_{j}}\right)\right] .
\end{aligned}
$$

Let us recall that we have

$$
\left(\overline{\bar{R}_{0, x_{j+1}}^{j} \overline{\bar{U}}_{1}^{j}}\right)=\exp \left[i \sum_{x \in B\left(x_{j+1}\right)} L^{-d} \frac{1}{i} \log \left(\bar{R}_{0, x_{j+1}}^{j} \overline{\bar{U}}_{1}^{j}\right)\left(\Gamma_{x_{j+1}, x}\right)\right] .
$$

The Eq. (104) can be written also in the following way:

$$
u(x)=\prod_{j=k-1}^{0}\left(R\left(U_{0}\left(\Gamma_{x_{j+1}, x}^{(j+1)}\right)\right)\right)^{-1}\left[\left(\overline{\bar{R}_{0, x_{j}+1}^{j} \overline{\bar{U}}_{1}^{j}}\right)^{-1}\left(\bar{R}_{0, x_{j}+1}^{j} \bar{U}_{1}^{j}\right)\left(\Gamma_{x_{j+1}, x_{j}}\right)\right],
$$

and this gives the formulas

$$
\begin{aligned}
&\left(\overline{R_{0} u}\right)^{j}\left(x_{j}\right)= \prod_{l=k-1}^{j}\left(R\left(\bar{U}_{0}^{j}\left(\Gamma_{x_{l+1}, x_{j}}^{(l+1-j)}\right)\right)\right)^{-1} \\
& \cdot\left[\left(\bar{R}_{0, x_{l+1}}^{l} \overline{\bar{U}}_{1}^{l}\right)^{-1}\left(\bar{R}_{0, x_{l+1}}^{l} \overline{\bar{U}}_{1}^{l}\right)\left(\Gamma_{x_{l+1}, x}\right)\right], \\
&\left(\overline{R_{0} u^{j}}\right)^{-1}\left(x_{j+1}\right) R\left(\bar{U}_{0}^{j}\left(\Gamma_{x_{J+1}, x_{\jmath}}\right)\right)\left(\overline{R_{0} u^{j}}\right)\left(x_{j}\right)=\left(\bar{R}_{0, x_{j+1}}^{j} \overline{\bar{U}}_{1}^{j}\right)\left(\Gamma_{x_{j+1}, x_{j}}\right) .
\end{aligned}
$$

\section{Properties of the Averages $\bar{U}_{1}^{k}$}

We will now investigate the new averaging operations. For the averages $\bar{U}^{k}$ natural quantities to consider were plaquette variables. For $\bar{U}_{1}^{k}$ we expect that the configuration itself, i.e., bond variables, has good bounds in terms of bounds of $U_{1}$. We will prove to this effect propositions analogous to Propositions 1 and 2. 
Let us recall that the definition of $\left(\bar{U}_{1}^{k}\right)_{c}, c \subset \Omega^{(k)}$, involves only the gauge fields $U_{1, b}, U_{0, b}$ at bonds $b \subset B^{k}\left(c_{-}\right) \cup B^{k}\left(c_{+}\right)$.

Let us start with a detailed analysis of the one-step averaging operation $\bar{V}_{1}$. We assume that the configurations $V_{0}, V_{1}$ satisfy the conditions

$$
\left|V_{0}(\partial p)-1\right|<\alpha_{0}, V_{1, b}=e^{i A_{b}},\left|A_{b}\right|<\alpha_{1}, \quad p, b \subset \Omega^{\prime},
$$

and $A_{b}$ belong to the complexified Lie algebra $\mathrm{g}^{c}$.

We would like to prove that for $\alpha_{0}, \alpha_{1}$ sufficiently small the average $\left(\bar{V}_{1}\right)_{c}$ is an analytic function of the variables $A_{b}, b \subset B\left(c_{-}\right) \cup B\left(c_{+}\right), c \subset \Omega^{\prime(1)}$, and to find bounds for $\left(\bar{V}_{1}\right)_{c}-1$, or rather $\frac{1}{i} \log \left(\bar{V}_{1}\right)_{c}$. We assume that $\alpha_{0}$ is so small that the Propositions 1 and 2 hold, i.e., $\alpha_{0} \leqq c_{2}$.

At first let us consider the expressions

$$
\overline{R_{0, y} V_{1}}=\exp \left[i \sum_{x \in B(y)} L^{-d} \frac{1}{i} \log \left(R_{0, y} V_{1}\right)\left(\Gamma_{y, x}\right)\right], \quad y \in \Omega^{(1)} .
$$

We have

$$
\frac{1}{i} \log \left(R_{0, y} V_{1}\right)\left(\Gamma_{y, x}\right)=\left(R_{0, y} A\right)\left(\Gamma_{y, x}\right)+O\left(\left(|A|\left(\Gamma_{y, x}\right)\right)^{2}\right),
$$

hence

$$
\overline{R_{0, y} V_{1}}=\exp \left[i \sum_{x \in B(y)} L^{-d}\left(R_{0, y} A\right)\left(\Gamma_{y, x}\right)+O\left(L^{2} \alpha_{1}^{2}\right)\right] .
$$

Next let us consider $\tilde{V}_{1}$ :

$$
\begin{aligned}
\left(\tilde{V}_{1}\right)_{c}= & \left(\overline{V_{1} V_{0}}\right)_{c}\left(\bar{V}_{0}\right)_{c}^{-1}=\exp \left[i \sum_{x \in B\left(c_{-}\right)} L^{-d} \frac{1}{i} \log \left(V_{1} V_{0}\right)\left(\Gamma_{c, x} \cup(-c)\right)\right] \\
& \cdot\left(V_{1} V_{0}\right)(c) V_{0}(c)^{-1} \exp \left[-i \sum_{x \in B\left(c_{-}\right)} L^{-d} \frac{1}{i} \log V_{0}\left(\Gamma_{c, x} \cup(-c)\right)\right] \\
= & \exp \left[i \sum_{x \in B\left(c_{-}\right)} L^{-d} \frac{1}{i} \log \left(R_{0, c_{-}} V_{1}\right)\left(\Gamma_{c, x} \cup(-c)\right) V_{0}\left(\Gamma_{c, x} \cup(-c)\right)\right] \\
& \cdot\left(R_{0, c_{-}} V_{1}\right)(c) \exp \left[-i \sum_{x \in B\left(c_{-}\right)} L^{-d} \frac{1}{i} \log V_{0}\left(\Gamma_{c, x} \cup(-c)\right)\right]
\end{aligned}
$$

and let us denote

$$
A_{x}=\frac{1}{i} \log \left(R_{0, c_{-}} V_{1}\right)\left(\Gamma_{c, x} \cup(-c)\right), Y_{x}=\frac{1}{i} \log V_{0}\left(\Gamma_{c, x} \cup(-c)\right) .
$$

As in (111) we have

$$
\begin{aligned}
A_{x}= & \left(R_{0, c_{-}} A\right)\left(\Gamma_{c, x} \cup(-c)\right)+O\left(L^{2} \alpha_{1}^{2}\right)=\left(R_{0, c_{-}} A\right)\left(\Gamma_{c_{-}, x} \cup\left[x, x^{\prime}\right]\right) \\
& -R\left(e^{i Y_{x}}\right)\left(R_{0, c_{-}} A\right)\left(c \cup \Gamma_{c_{+}, x^{\prime}}\right)+O\left(L^{2} \alpha_{1}^{2}\right) .
\end{aligned}
$$

A logarithm in the first exponential in (113) can be written as $\frac{1}{i} \log e^{i A_{x}} e^{i Y_{x}}$. 
Applying the results of Sect. A, more exactly (38), we have

$$
\frac{1}{i} \log e^{i A_{x}} e^{i Y_{x}}=Y_{x}+g^{-1}\left(-i \operatorname{ad}_{Y_{x}}\right) A_{x}+O\left(\left|A_{x}\right|^{2}\right) .
$$

All these formulas give us

$$
\begin{aligned}
\left(\tilde{V}_{1}\right)_{c}= & \exp \left[i \sum_{x \in B\left(c_{-}\right)} L^{-d} Y_{x}+i \sum_{x \in B\left(c_{-}\right)} L^{-d} g^{-1}\left(-i \operatorname{ad}_{Y_{x}}\right) A_{x}+O\left(L^{2} \alpha_{1}^{2}\right)\right] \\
& \cdot \exp \left[i\left(R_{0, c_{-}} A\right)(c)+O\left(L^{2} \alpha_{1}^{2}\right)\right] \exp \left[-i \sum_{x \in B\left(c_{-}\right)} L^{-d} Y_{x}\right] .
\end{aligned}
$$

Denoting $\sum_{x \in B\left(c_{-}\right)} L^{-d} Y_{x}=Y$ and using the formula (41), we have further

where

$$
\begin{aligned}
\left(\tilde{V}_{1}\right)_{c}= & \exp \left[i \sum_{x \in B\left(c_{-}\right)} L^{-d} g^{-1}\left(-i \operatorname{ad}_{Y_{x}}\right) A_{x}+O\left(L^{2} \alpha_{1}^{2}\right)+i Y\right] \\
& \cdot \exp [-i Y] \exp \left[i R\left(e^{i Y}\right)\left(R_{0_{,-}-} A\right)(c)+O\left(L^{2} \alpha_{1}^{2}\right)\right] \\
= & \exp \left[i g\left(-i \operatorname{ad}_{Y}\right) \sum_{x \in B\left(c_{-}\right)} L^{-d} g^{-1}\left(-i \operatorname{ad}_{Y_{x}}\right) A_{x}+O\left(L^{2} \alpha_{1}^{2}\right)\right] \\
& \cdot \exp \left[i R\left(e^{i Y}\right)\left(R_{0, c_{-}} A\right)(c)+O\left(L^{2} \alpha_{1}^{2}\right)\right] \\
= & \exp \left[\left(Q^{\prime}\left(V_{0}\right) A\right)_{c}+O\left(L^{2} \alpha_{1}^{2}\right)\right],
\end{aligned}
$$

$$
\begin{aligned}
\left(Q^{\prime}\left(V_{0}\right) A\right)_{c}= & g\left(-i \operatorname{ad}_{Y}\right) \sum_{x \in B\left(c_{-}\right)} L^{-d} g^{-1}\left(-i \operatorname{ad}_{Y_{x}}\right) \\
& \cdot\left[\left(R_{0, c_{-}} A\right)\left(\Gamma_{c_{-}, x} \cup\left[x, x^{\prime}\right]\right)-R\left(e^{i Y_{x}}\right)\left(R_{0, c_{-}} A\right)\left(c \cup \Gamma_{c_{+}, x^{\prime}}\right)\right] \\
& +R\left(e^{i Y}\right)\left(R_{0, c_{-}} A\right)(c),
\end{aligned}
$$

as it follows from (115).

We can transform this linear expression using the identities $R\left(e^{i Y_{x}}\right)=e^{i \operatorname{ad}_{y_{x}}}$ and $g^{-1}\left(-i \operatorname{ad}_{Y_{x}}\right) e^{i \operatorname{ad}_{Y_{x}}}=g^{-1}\left(i \operatorname{ad}_{Y_{x}}\right)$. The last follows from the corresponding identity for functions of a complex variable: $g^{-1}(-z) e^{z}=g^{-1}(z)$. We have also

$$
\begin{aligned}
\left(R_{0, c_{-}} A\right)\left(\Gamma_{c_{+}, x^{\prime}}\right) & =R\left(V_{0}(c)\right)\left(R_{0, c_{+}} A\right)\left(\Gamma_{c_{+}, x^{\prime}}\right) \\
& =R\left(e^{-i Y}\right) R\left(\left(\bar{V}_{0}\right)_{c}\right)\left(R_{0, c_{+}} A\right)\left(\Gamma_{c_{+}, x^{\prime}}\right) \\
& =e^{-i \operatorname{ad}_{Y}} \bar{R}_{0, c}\left(R_{0, c_{+}} A\right)\left(\Gamma_{c_{+}, x^{\prime}}\right) .
\end{aligned}
$$

These identities imply the following formula:

$$
\begin{aligned}
\left(Q^{\prime}\left(V_{0}\right) A\right)_{c}= & g\left(-i \operatorname{ad}_{Y}\right) \sum_{x \in B\left(c_{-}\right)} L^{-d} g^{-1}\left(-i \operatorname{ad}_{Y_{x}}\right)\left(R_{0, c_{-}} A\right)\left(\Gamma_{c_{-}, x} \cup\left[x, x^{\prime}\right]\right) \\
& -g\left(-i \operatorname{ad}_{Y}\right) \sum_{x \in B\left(c_{-}\right)} L^{-d} g^{-1}\left(i \operatorname{ad}_{Y_{x}}\right) e^{-i \operatorname{ad}_{Y}} \bar{R}_{0, c}\left(R_{0, c_{+}} A\right)\left(\Gamma_{c_{+}, x^{\prime}}\right) \\
& +\left[e^{i \mathrm{ad}_{Y}}-g\left(-i \operatorname{ad}_{Y}\right) \sum_{x \in B\left(c_{-}\right)} L^{-d} g^{-1}\left(i \operatorname{ad}_{Y_{x}}\right)\right]\left(R_{0, c_{-}} A\right)(c) .
\end{aligned}
$$

From (118) and (112) we get finally

$$
\begin{aligned}
\left(\bar{V}_{1}\right)_{c}= & \left(\overline{R_{0, c_{-}} V_{1}}\right)^{-1}\left(\tilde{V}_{1}\right)_{c} \bar{R}_{0, c} \overline{R_{0, c_{+}} V_{1}} \\
= & \exp \left[-i \sum_{x \in B\left(c_{-}\right)} L^{-d}\left(R_{0, c_{-}} A\right)\left(\Gamma_{c_{-}, x}\right)+i\left(Q^{\prime}\left(V_{0}\right) A\right)_{c}\right. \\
& \left.+i \sum_{x^{\prime} \in B\left(c_{+}\right)} L^{-d} \bar{R}_{0, c}\left(R_{0, c_{+}} A\right)\left(\Gamma_{c_{+}, x^{\prime}}\right)+O\left(L^{2} \alpha_{1}^{2}\right)\right] .
\end{aligned}
$$


In all the estimates above, we have assumed that $\alpha_{0}, \alpha_{1}$ are sufficiently small so that the formulas and inequalities proved in Sect. A hold, and that $\left|Y_{x}\right|=O\left(L^{2} \alpha_{0}\right)$ are small. We restrict further $\alpha_{1}$ assuming that the norm of the element in the exponential above is small also; for example, it is enough that it is $\leqq \frac{1}{2}$. Under these assumptions it is obvious that functions of the variables $A$ involved in all the formulas until now are analytic functions of $A$. Let us define

$$
Q\left(V_{0}, A, c\right)=\frac{1}{i} \log \left(\bar{V}_{1}\right)_{c},
$$

then $Q\left(V_{0}, A, c\right)$ is an analytic function of $A$ and from (120) it follows that its Taylor expansion begins with a first-order polynomial. Let us denote it by $L\left(Q\left(V_{0}\right) A\right)_{c}$. Thus we have

$$
Q\left(V_{0}, A, c\right)=L\left(Q\left(V_{0}\right) A\right)_{c}+C\left(V_{0}, A, c\right) .
$$

$C\left(V_{0}, A, c\right)$ is an analytic function of $A$ whose Taylor's expansion begins with a second-order polynomial (a quadratic form), and

$$
\left|C\left(V_{0}, A, c\right)\right| \leqq C_{1} L^{2}|A|^{2}<C_{1}\left(L \alpha_{1}\right)^{2} .
$$

The linear form $Q\left(V_{0}\right) A$ is given by

$$
\begin{aligned}
& \left(Q\left(V_{0}\right) A\right)_{c}=\sum_{x \in B\left(c_{-}\right)} L^{-(d+1)}\left(R_{0, c_{-}} A\right)\left(\left[x, x^{\prime}\right]\right)+\sum_{x \in B\left(c_{-}\right)} L^{-(d+1)} \\
& \cdot\left[g\left(-i \operatorname{ad}_{Y}\right) g^{-1}\left(-i \operatorname{ad}_{Y_{x}}\right)-1\right]\left(R_{0, c_{-}} A\right)\left(\Gamma_{c_{-}, x}\right) \\
& +\sum_{x \in B\left(c_{-}\right)} L^{-(d+1)}\left[g\left(-i \operatorname{ad}_{Y}\right) g^{-1}\left(-i \operatorname{ad}_{Y_{x}}\right)-1\right] \\
& \cdot\left(R_{0, c_{-}} A\right)\left(\left[x, x^{\prime}\right]\right)-\sum_{x \in B\left(c_{-}\right)} L^{-(d+1)} \\
& \cdot\left[g\left(-i \operatorname{ad}_{Y}\right) g^{-1}\left(i \operatorname{ad}_{Y_{x}}\right) e^{-i \operatorname{ad}_{Y}}-1\right] \bar{R}_{0, c}\left(R_{0, c_{+}} A\right)\left(\Gamma_{c_{+}, x^{\prime}}\right) \\
& +\left[e^{i \operatorname{ad}_{Y}}-g\left(-i \operatorname{ad}_{Y}\right) \sum_{x \in B\left(c_{-}\right)} L^{-d} g^{-1}\left(i \operatorname{ad}_{Y_{x}}\right)\right] L^{-1}\left(R_{0, c_{-}} A\right)(c) .
\end{aligned}
$$

The first term on the right-hand side above is the main term in this linear form, and it resembles the definition of the averaging operation $Q$ in [2]. The remaining terms are small because the functions $g(-z), g^{-1}(z), e^{i z}$ are equal to 1 for $z=0$, so the operators occurring in these terms can be estimated by $O\left(L^{2} \alpha_{0}\right)$ and the terms can be estimated by $O(1) L^{2} \alpha_{0} L|A|<O(1) L^{2} \alpha_{0} L \alpha_{1}$. We will denote the main term by $Q_{V_{0}}$, or $Q_{0}$

$$
\left(Q_{0} A\right)_{c}=\left(Q_{V_{0}} A\right)_{c}=\sum_{x \in B(c-)} L^{-(d+1)}\left(R_{0, c_{-}} A\right)\left(\left[x, x^{\prime}\right]\right),
$$

and it has an estimate

$$
\left|\left(Q_{0} A\right)_{c}\right| \leqq|A|<\alpha_{1},
$$

so we have for the whole linear term

$$
\left|\left(Q\left(V_{0}\right) A\right)_{c}\right| \leqq|A|+O(1) L^{2} \alpha_{0}|A|<\left(1+O(1) L^{2} \alpha_{0}\right) \alpha_{1}<e^{O(1) L^{2} \alpha_{0}} \alpha_{1} .
$$

Thus we have proved the following

Proposition 3. There exist constants $C_{1}, c_{3}, c_{3} \leqq c_{2}$, such that for $\alpha_{0}, \alpha_{1} \leqq c_{3}$ the function $Q\left(V_{0}, A\right)=\frac{1}{i} \log \overline{\bar{V}}_{1}$ is an analytic function of $A$ satisfying the equalities and bounds (122)-(124). The constant $C_{1}$ depends on $d$ and $c_{3}$ depends on $d$ and $L$. 
Now we will consider the $k^{\text {th }}$ order average $\bar{U}_{1}^{k}$. Its definition implies that $\frac{1}{i} \log \bar{U}_{1}^{k}$ as a function of $\frac{1}{i} \log U_{1}$ is a composition of the functions

$$
Q\left(U_{0}, \cdot\right), Q\left(\bar{U}_{0}, \cdot\right), \ldots, Q\left(\bar{U}_{0}^{k-2}, \cdot\right), Q\left(\bar{U}_{0}^{k-1}, \cdot\right) .
$$

We assume that $U_{0}$ satisfies the assumptions of Proposition 2 and $U_{1}=e^{i \eta A}$, $|A|<\alpha_{1}$. Then by this proposition the configurations $\bar{U}_{0}^{j}$ for $j<k$ satisfy the assumptions of Proposition 3 for $V_{0}=\bar{U}_{0}^{j}$ if $\alpha_{0} L^{2 j} \eta^{2}+2 C_{0}\left(\alpha_{0} L^{2 j} \eta^{2}\right)^{2}$ $\leqq 2 \alpha_{0} L^{2 j} \eta^{2}<\alpha_{0} \leqq c_{3}$.

We will now investigate compositions of functions in the sequence (127). For the first function we have

$$
\left|Q\left(U_{0}, \eta A\right)-L \eta Q\left(U_{0}\right) A\right| \leqq C_{1}(L|\eta A|)^{2}<C_{1}\left(\alpha_{1} L \eta\right)^{2},
$$

hence

$$
\left|\frac{1}{L \eta} Q\left(U_{0}, \eta A\right)-Q\left(U_{0}\right) A\right|<C_{1} L \eta \alpha_{1}^{2},
$$

and

$$
\begin{aligned}
\left|\frac{1}{L \eta} Q\left(U_{0}, \eta A\right)\right| & <\left|Q\left(U_{0}\right) A\right|+C_{1} L \eta \alpha_{1}^{2}<\left(1+O(1) L^{2} \alpha_{0} \eta^{2}\right) \alpha_{1}+C_{1} L \eta \alpha_{1}^{2} \\
& <e^{O(1) L^{2} \eta^{2} \alpha_{0}} \alpha_{1}+C_{1} L \eta \alpha_{1}^{2} .
\end{aligned}
$$

Because $e^{O(1) L^{2} \eta^{2} \alpha_{0}} \alpha_{1}+C_{1} L \eta \alpha_{1}^{2} \leqq e^{O(1) \alpha_{0}} \alpha_{1}+C_{1} \alpha_{1}^{2} \leqq 2 \alpha_{1}$ for $\alpha_{0}, \alpha_{1}$ sufficiently small $\left(O(1) \alpha_{0} \leqq \frac{1}{3}, C_{1} \alpha_{1} \leqq \frac{1}{2}\right)$, so $\left|Q\left(U_{0}, \eta A\right)\right|<2 \alpha_{1} L \eta \leqq 2 \alpha_{1} \leqq c_{3}$ for $\alpha_{1} \leqq \frac{1}{2} c_{3}$, and we can apply Proposition 3 to function $Q\left(\bar{U}_{0}, \cdot\right)$ calculated at $Q\left(U_{0}, \eta A\right)$. For this composition we have

denoting

$$
\begin{aligned}
& \left|Q\left(\bar{U}_{0}, Q\left(U_{0}, \eta A\right)\right)-L^{2} \eta Q\left(\bar{U}_{0}\right) \frac{1}{L \eta} Q\left(U_{0}, \eta A\right)\right| \\
& \quad \leqq C_{1}\left(L^{2} \eta\right)^{2}\left|\frac{1}{L \eta} Q\left(U_{0}, \eta A\right)\right|^{2}<C_{1}\left(L^{2} \eta\right)^{2}\left(2 \alpha_{1}\right)^{2},
\end{aligned}
$$

$$
Q\left(\bar{U}_{0}, Q\left(U_{0}, \eta A\right)\right)=Q_{2}\left(U_{0}, \eta A\right), Q\left(\bar{U}_{0}\right) Q\left(U_{0}\right)=Q_{2}\left(U_{0}\right),
$$

and using (128) we get

$$
\begin{aligned}
\left|\frac{1}{L^{2} \eta} Q_{2}\left(U_{0}, \eta A\right)-Q_{2}\left(U_{0}\right) A\right| & <e^{O(1) L^{4} \eta^{2} \alpha_{0}} C_{1} L \eta \alpha_{1}^{2}+4 C_{1} L^{2} \eta \alpha_{1}^{2} \\
& <e^{O(1)\left(L^{4}+L^{2}\right) \eta^{2} \alpha_{0}} 4 C_{1}\left(L+L^{2}\right) \eta \alpha_{1}^{2} \\
\left|\frac{1}{L^{2} \eta} Q_{2}\left(U_{0}, \eta A\right)\right| & <e^{O(1)\left(L^{4}+L^{2}\right) \eta^{2} \alpha_{0}} \alpha_{1} \\
& +e^{O(1)\left(L^{4}+L^{2}\right) \eta^{2} \alpha_{0}} 4 C_{1}\left(L+L^{2}\right) \eta \alpha_{1}^{2} \\
& <e^{O(1)\left(1+L^{-2}\right) \alpha_{0}}\left(1+4 C_{1}\left(1+L^{-1}\right) \alpha_{1}\right) \alpha_{1} \\
& <e^{O(1) 2 \alpha_{0}}\left(1+8 C_{1} \alpha_{1}\right) \alpha_{1} \\
& <2 \alpha_{1} \quad \text { for } \alpha_{0}, \alpha_{1} \text { sufficiently small [e.g., } O(1) \alpha_{0} \leqq \frac{1}{6}, \\
& \left.\quad 8 C_{1} \alpha_{1} \leqq \frac{1}{3}\right] .
\end{aligned}
$$


Continuing these arguments, we arrive at the following inductive assumption for the composition $Q_{j}\left(U_{0}, \eta A\right)$ of the first $j$ functions (127) aad the composition $Q_{j}\left(U_{0}\right)$ of their linear parts:

$$
\left|\frac{1}{L^{j} \eta} Q_{j}\left(U_{0}, \eta A\right)-Q_{j}\left(U_{0}\right) A\right|<e^{O(1)\left(L^{2 j}+\ldots+L^{4}+L^{2}\right) \eta^{2} \alpha_{0}} \cdot 4 C_{1}\left(L^{j}+\ldots+L^{2}+L\right) \eta \alpha_{1}^{2} .
$$

This implies

$$
\begin{aligned}
\left|\frac{1}{L^{j} \eta} Q_{j}\left(U_{0}, \eta A\right)\right| & <e^{O(1)\left(L^{2 j}+\ldots+L^{4}+L^{2}\right) \eta^{2} \alpha_{0}}\left(\alpha_{1}+4 C_{1}\left(L^{j}+\ldots+L^{2}+L\right) \eta \alpha_{1}^{2}\right) \\
& \leqq e^{O(1)\left(1+L^{-2}+\ldots+L^{-2(j-1)}\right) \alpha_{0}}\left(\alpha_{1}+4 C_{1}\left(1+L^{-1}+\ldots+L^{-(j-1)}\right) \alpha_{1}^{2}\right) \\
& <e^{O(1) 2 \alpha_{0}}\left(1+8 C_{1} \alpha_{1}\right) \alpha_{1}<2 \alpha_{1},
\end{aligned}
$$

and for $\alpha_{1} \leqq \frac{1}{2} c_{3}$ we can apply Proposition 3 to the function $Q\left(\bar{U}_{0}^{j}, \cdot\right)$ calculated at $Q_{j}\left(U_{0}, \eta A\right)$, and we get

$$
\begin{aligned}
& \left|Q\left(\bar{U}_{0}^{j}, Q_{j}\left(U_{0}, \eta A\right)\right)-L^{j+1} \eta Q\left(\bar{U}_{0}^{j}\right) \frac{1}{L^{j} \eta} Q_{j}\left(U_{0}, \eta A\right)\right| \\
& \quad<C_{1}\left(L^{j+1} \eta\right)^{2}\left|\frac{1}{L^{j} \eta} Q_{j}\left(U_{0}, \eta A\right)\right|^{2}<4 C_{1}\left(L^{j+1} \eta\right)^{2} \alpha_{1}^{2} .
\end{aligned}
$$

Applying (130) and denoting

$$
Q_{j+1}\left(U_{0}, \eta A\right)=Q\left(\bar{U}_{0}^{j}, Q_{j}\left(U_{0}, \eta A\right)\right), Q_{j+1}\left(U_{0}\right)=Q\left(\bar{U}_{0}^{j}\right) Q_{j}\left(U_{0}\right),
$$

we have

$$
\begin{aligned}
\left|\frac{1}{L^{j+1} \eta} Q_{j+1}\left(U_{0}, \eta A\right)-Q_{j+1}\left(U_{0}\right) A\right|< & 4 C_{1} L^{j+1} \eta \alpha_{1}^{2}+e^{O(1) L^{2} L^{2 j} \eta^{2} \alpha_{0}} \\
& \cdot e^{O(1)\left(L^{2 j}+\ldots+L^{2}\right) \eta^{2} \alpha_{0}} 4 C_{1}\left(L^{j}+\ldots+L\right) \eta \alpha_{1}^{2} \\
< & e^{O(1)\left(L^{2(j+1)}+L^{2 j}+\ldots+L^{2}\right) \eta^{2} \alpha_{0}} 4 C_{1}\left(L^{j+1}\right. \\
& \left.+L^{j}+\ldots+L\right) \eta \alpha_{1}^{2} .
\end{aligned}
$$

Thus the inductive hypothesis (130) is proved for $j \leqq k$. For $j=k$, we have

$$
\begin{aligned}
& \left|Q_{k}\left(U_{0}, \eta A\right)-Q_{k}\left(U_{0}\right) A\right|<e^{O(1)\left(1+L^{-2}+\ldots+L^{-2(k-1)) \alpha_{0}}\right.} \\
& \quad \cdot 4 C_{1}\left(1+L^{-1}+\ldots+L^{-(k-1)}\right) \alpha_{1}^{2}<e^{O(1) 2 \alpha_{0}} 8 C_{1} \alpha_{1}^{2}=C_{2} \alpha_{1}^{2} .
\end{aligned}
$$

We formulate the obtained results in

Proposition 4. There exist constants $C_{2}, c_{4}$ such that for $\alpha_{0}, \alpha_{1} \leqq c_{4}$ the function $Q_{k}\left(U_{0}, \eta A, c\right)=\frac{1}{i} \log \left(\bar{U}_{1}^{k}\right)_{c}, c \subset \Omega^{(k)}$, is an analytic function of the variables $A_{b}, b$ $C B^{k}\left(c_{-}\right) \cup B^{k}\left(c_{+}\right)$. Further we have

$$
Q_{k}\left(U_{0}, \eta A\right)=Q_{k}\left(U_{0}\right) A+C_{k}\left(U_{0}, A\right),
$$

and

$$
\left|C_{k}\left(U_{0}, A\right)\right| \leqq C_{2}|A|^{2}<C_{2} \alpha_{1}^{2}
$$


The constants $C_{2}, c_{4}$ are independent of $k, C_{2}$ depends ond and $c_{4}$ depends on $d$ and $L$.

The function $C_{k}$ can be decomposed further into a sum of homogeneous polynomials,

$$
C_{k}\left(U_{0}, A\right)=C_{k}^{(2)}\left(U_{0}, A\right)+C_{k}^{(3)}\left(U_{0}, A\right)+\ldots
$$

We will need some more precise information about the function $Q_{k}$. This information is connected with a notion of the functional derivative. Let us recall this notion. If $F(A)$ is a differentiable function defined at field configurations $A$ on $\Omega$, then the differential

$$
d F(A, \delta A)=\left.\frac{d}{d t} F(A+t \delta A)\right|_{t=0}
$$

is a linear functional of the variable $\delta A$ and can be represented as a scalar product of $\delta A$ and some Lie algebra valued function. This function is called the functional derivative and is denoted by $\frac{\delta}{\delta A} F(A)$, thus we have

$$
\left.\frac{d}{d t} F(A+t \delta A)\right|_{t=0}=\sum_{b \subset \Omega} \eta^{d} \operatorname{tr} \frac{\delta F(A)}{\delta A_{b}} \delta A_{b}=\left\langle\frac{\delta F(A)}{\delta A}, \delta A\right\rangle .
$$

From this definition it follows easily that the functional derivative coincides with partial derivatives (gradient) of $F(A)$ multiplied by $\eta^{-d}$.

We would like to prove that the functional derivative of $Q_{k}\left(U_{0}, \eta A\right)$ is bounded by a constant independent of $\eta$. This property is not clear even for the linear part of $Q_{k}$, so let us start with an analysis of this linear part. The linear part of the one-step renormalization transformation is given by the formula (124). It is a sum of the main term $Q_{V_{0}} A$ given by (125) and a remainder which we will denote by $Q^{\prime \prime}\left(V_{0}\right) A$. From (124) it is clear that we have the inequalities

$$
\left|Q_{V_{0}} A\right| \leqq Q|A|,\left|Q^{\prime \prime}\left(V_{0}\right) A\right| \leqq C_{1}^{\prime} L^{2} \alpha_{0} Q^{\prime \prime}|A|,
$$

where the operator $Q$ is defined as in [2], and $Q^{\prime \prime}$ is defined as

$$
\left(Q^{\prime \prime} A\right)_{c}=\sum_{b \subset B(c-) \cup B\left(c_{+}\right)} L^{-d} A_{b} .
$$

The constant $C_{1}^{\prime}$ depends on $d$ and $L$. A composition of $k$ operators $Q$ is the operator $Q_{k}$. The operators $Q^{\prime \prime}$ do not compose in a simple way, but if we introduce an operator $Q_{k}^{\prime \prime}$ by the formula

$$
\left(Q_{k}^{\prime \prime} A\right)_{c}=\sum_{b \subset B^{k}(c-) \cup B^{k}\left(c_{+}\right)} \eta^{d} A_{b}, \quad c \subset \Omega^{(k)},
$$

then we have the inequality

$$
\left|Q^{\prime \prime} Q_{j}^{\prime \prime} A\right| \leqq Q^{\prime \prime} Q_{j}^{\prime \prime}|A| \leqq 2 d Q_{j+1}^{\prime \prime}|A| .
$$

Now we will prove by induction the bound

$$
\left|Q_{j}\left(U_{0}\right) A\right| \leqq Q_{j}|A|+2 C_{1}^{\prime} \alpha_{0}\left(L^{j} \eta\right)^{2} Q_{j}^{\prime \prime}|A|, \quad j \leqq k,
$$


assuming that $U_{0}$ satisfies the bound (52). For $j=1$ it is a consequence of (139). We assume (143) for $j<k$ and we have

$$
\begin{aligned}
\left|Q_{j+1}\left(U_{0}\right) A\right|= & \left|Q\left(\bar{U}_{0}^{j}\right) Q_{j}\left(U_{0}\right) A\right| \leqq Q\left|Q_{j}\left(U_{0}\right) A\right|+C_{1}^{\prime} 2 \alpha_{0}\left(L^{j} \eta\right)^{2} Q^{\prime \prime}\left|Q_{j}\left(U_{0}\right) A\right| \\
\leqq & Q\left(Q_{j}|A|+2 C_{1}^{\prime} \alpha_{0}\left(L^{j} \eta\right)^{2} Q_{j}^{\prime \prime}|A|\right)+C_{1}^{\prime} 2 \alpha_{0}\left(L^{j} \eta\right)^{2} \\
& \cdot Q^{\prime \prime}\left(Q_{j}|A|+2 C_{1}^{\prime} \alpha_{0}\left(L^{j} \eta\right)^{2} Q_{j}^{\prime \prime}|A|\right) \\
= & Q_{j+1}|A|+2 C_{1}^{\prime} \alpha_{0}\left(L^{j} \eta\right)^{2} Q Q_{j}^{\prime \prime}|A|+2 C_{1}^{\prime} \alpha_{0}\left(L^{j} \eta\right)^{2} Q^{\prime \prime} Q_{j}|A| \\
& +4 C_{1}^{\prime 2} \alpha_{0}^{2}\left(L^{j} \eta\right)^{4} Q^{\prime \prime} Q_{j}^{\prime \prime}|A|
\end{aligned}
$$

by (139) and Proposition 2. Further using the inequalities (142), $Q Q_{j}^{\prime \prime}|A|$ $\leqq 2 Q_{j+1}^{\prime \prime}|A|$, and $Q^{\prime \prime} Q_{j}|A| \leqq Q_{j+1}^{\prime \prime}|A|$, we get

$$
\left|Q_{j+1}\left(U_{0}\right) A\right| \leqq Q_{j+1}|A|+2 C_{1}^{\prime} \alpha_{0}\left(L^{j+1} \eta\right)^{2} Q_{j+1}^{\prime \prime}|A| \cdot\left(2 L^{-2}+L^{-2}+4 d C_{1}^{\prime} \alpha_{0} L^{-4}\right) .
$$

Because

$$
2 L^{-2}+L^{-2}+4 d C_{1}^{\prime} \alpha_{0} L^{-4} \leqq \frac{3}{4}+4 d C_{1}^{\prime} L^{-4} \alpha_{0} \leqq 1
$$

if $16 d C_{1}^{\prime} L^{-4} \alpha_{0} \leqq 1$, so the bound (143) is proved for all $j \leqq k$. For $j=k$ we have

$$
\left|Q_{k}\left(U_{0}\right) A\right| \leqq Q_{k}|A|+2 C_{1}^{\prime} \alpha_{0} Q_{k}^{\prime \prime}|A| \leqq\left(1+2 C_{1}^{\prime} \alpha_{0}\right) Q_{k}^{\prime \prime}|A|,
$$

and this bound implies the required property, namely

$$
\frac{\delta}{\delta A_{b}}\left(Q_{k}\left(U_{0}\right) A\right)_{c}=Q_{k}\left(U_{0} ; c, b\right),\left|Q_{k}\left(U_{0} ; c, b\right)\right| \leqq 1+2 C_{1}^{\prime} \alpha_{0} .
$$

Now we will generalize it to the whole function $Q_{k}$. It is enough to prove it for $C_{k}$. For one-step renormalization transformation we have the bound

$$
\left|\left\langle\frac{\delta C\left(V_{0}, A\right)}{\delta A}, \delta A\right\rangle\right| \leqq C_{1}^{\prime \prime}|A| Q^{\prime \prime}|\delta A|
$$

following easily from general properties of the function $C\left(V_{0}, A\right)$. The constant $C_{1}^{\prime \prime}$ depends on $d$ and $L$. We will prove that a similar bound holds for the functional derivative of $C_{j}\left(U_{0}, A\right)$ for arbitrary $j \leqq k$. We will prove by induction that

$$
\left|\left\langle\frac{\delta}{\delta A} C_{j}\left(U_{0}, A\right), \delta A\right\rangle\right| \leqq C_{3}|A| Q_{j}^{\prime \prime}|\delta A|,
$$

where the configurations $A$ are considered on $L^{-j}$-lattice and $C_{3}$ is a positive constant satisfying conditions which will be written later.

From the definition of the functions $Q_{j}\left(U_{0}, \eta A\right)$ we have

$$
\begin{aligned}
Q_{j+1}\left(U_{0}, \eta A\right) & =Q\left(\bar{U}_{0}^{j}, Q_{j}\left(U_{0}, \eta A\right)\right), \\
Q_{j}\left(U_{0}, \eta A\right) & =L^{j} \eta Q_{j}\left(U_{0}\right) A+C_{j}\left(U_{0}, L^{j} \eta A\right),
\end{aligned}
$$

hence

$$
\begin{aligned}
Q_{j+1}\left(U_{0}, \eta A\right)= & L Q\left(\bar{U}_{0}^{j}\right) Q_{j}\left(U_{0}, \eta A\right)+C\left(\bar{U}_{0}^{j}, Q_{j}\left(U_{0}, \eta A\right)\right) \\
= & L^{j+1} \eta Q\left(\bar{U}_{0}^{j}\right) Q_{j}\left(U_{0}\right) A+L Q\left(\bar{U}_{0}^{j}\right) C_{j}\left(U_{0}, L^{j} \eta A\right) \\
& +C\left(\bar{U}_{0}^{j}, Q_{j}\left(U_{0}, \eta A\right)\right) \\
= & L^{j+1} \eta Q_{j+1}\left(U_{0}\right) A+C_{j+1}\left(U_{0}, L^{j+1} \eta A\right),
\end{aligned}
$$


and

$$
C_{j+1}\left(U_{0}, A\right)=L Q\left(\bar{U}_{0}^{j}\right) C_{j}\left(U_{0}, L^{-1} A\right)+C\left(\bar{U}_{0}^{j}, L^{-1} Q_{j}\left(U_{0}\right) A+C_{j}\left(U_{0}, L^{-1} A\right)\right),
$$

$A$ is a field configuration considered on $L^{-(j+1)} Z^{d},|A|<L^{j+1} \eta \alpha_{1}$. Differentiation of the above equality gives

$$
\begin{aligned}
\left\langle\frac{\delta}{\delta A} C_{j+1}\left(U_{0}, A\right), \delta A\right\rangle= & Q\left(\bar{U}_{0}^{j}\right)\left\langle\frac{\delta C_{j}}{\delta A}\left(U_{0}, L^{-1} A\right), \delta A\right\rangle \\
& +\left\langle\frac{\delta C}{\delta A}\left(\bar{U}_{0}^{j}, L^{-1} Q_{j}\left(U_{0}\right) A+C_{j}\left(U_{0}, L^{-1} A\right)\right), L^{-1} Q_{j}\left(U_{0}\right) \delta A\right. \\
& \left.+L^{-1}\left\langle\frac{\delta C_{j}}{\delta A}\left(U_{0}, L^{-1} A\right), \delta A\right\rangle\right\rangle .
\end{aligned}
$$

Using (143), (148), (149) we obtain the following bound:

$$
\begin{aligned}
\left|\left\langle\frac{\delta}{\delta A} C_{j+1}\left(U_{0}, A\right), \delta A\right\rangle\right| & Q C_{3} L^{-1}|A| Q_{j}^{\prime \prime}|\delta A| \\
& +C_{1}^{\prime} 2 \alpha_{0}\left(L^{j} \eta\right)^{2} Q^{\prime \prime} C_{3} L^{-1}|A| Q_{j}^{\prime \prime}|\delta A| \\
& +C_{1}^{\prime \prime}\left|L^{-1} Q_{j}\left(U_{0}\right) A+C_{j}\left(U_{0}, L^{-1} A\right)\right| \\
& \cdot Q^{\prime \prime}\left|L^{-1} Q_{j}\left(U_{0}\right) \delta A+L^{-1}\left\langle\frac{\delta C_{j}}{\delta A}\left(U_{0}, L^{-1} A\right), \delta A\right\rangle\right| \\
\leqq & C_{3}\left(2-L^{-1}\right) L^{-1}|A| Q_{j+1}^{\prime \prime}|\delta A| \\
& +C_{1}^{\prime} C_{3} 2 \alpha_{0} 2 d\left(L^{j} \eta\right)^{2} L^{-1}|A| Q_{j+1}^{\prime \prime}|\delta A| \\
& +C_{1}^{\prime \prime} L^{-2}\left(Q_{j}|A|+2 C_{1}^{\prime} \alpha_{0}\left(L^{j} \eta\right)^{2} Q_{j}^{\prime \prime}|A|+C_{2} L^{-1}|A|^{2}\right) \\
& \cdot Q^{\prime \prime}\left(Q_{j}|\delta A|+2 C_{1}^{\prime} \alpha_{0}\left(L^{j} \eta\right)^{2} Q_{j}^{\prime \prime}|\delta A|+C_{3} L^{-1}|A| Q_{j}^{\prime \prime}|\delta A|\right) \\
\leq & C_{3}|A| Q_{j+1}^{\prime \prime}|\delta A|\left[1-L^{-2}+4 d C_{1}^{\prime} \alpha_{0} L^{-3}\right] \\
& +C_{1}^{\prime \prime} L^{-2}\left(1+4 d C_{1}^{\prime} \alpha_{0} L^{-2}+C_{2} L^{-1} \alpha_{1}\right) \\
& \cdot|A|\left(1+4 d C_{1}^{\prime} \alpha_{0} L^{-2}+2 d C_{3} L^{-1} \alpha_{1}\right) Q_{j+1}^{\prime \prime}|\delta A| \\
\leqq & C_{3}|A| Q_{j+1}^{\prime \prime}|\delta A|\left[1-L^{-2}+4 d C_{1}^{\prime} \alpha_{0} L^{-3}+\frac{C_{1}^{\prime \prime} L^{-2}}{C_{3}}\right. \\
& \left.\cdot\left(1+4 d C_{1}^{\prime} \alpha_{0} L^{-2}+C_{2} L^{-1} \alpha_{1}+2 d C_{3} L^{-1} \alpha_{1}\right)^{2}\right] . \quad(1
\end{aligned}
$$

This bound implies the inequality (149) for $j+1$ if

$$
4 d C_{1}^{\prime} \alpha_{0} L^{-1}+\frac{C_{1}^{\prime \prime}}{C_{3}}\left(1+4 d C_{1}^{\prime} \alpha_{0} L^{-2}+C_{2} L^{-1} \alpha_{1}+2 d C_{3} L^{-1} \alpha_{1}\right)^{2} \leqq 1 .
$$

This inequality is satisfied if $C_{3}>C_{1}^{\prime \prime}$ and $\alpha_{0}, \alpha_{1}$ are sufficiently small, e.g., we may take $C_{3}=6 C_{1}^{\prime \prime}$ and $\alpha_{0}, \alpha_{1}$ satisfying $4 d C_{1}^{\prime} \alpha_{0} L^{-1} \leqq \frac{1}{3},\left(C_{2} L^{-1}+12 d C_{1}^{\prime \prime} L^{-1}\right) \alpha_{1} \leqq \frac{2}{3}$. Thus we have proved 
Proposition 5. The functional derivative of $Q_{k}\left(U_{0}, \eta A\right)$ is a bounded function for $\alpha_{0}$, $\alpha_{1}$ sufficiently small, and we have the bounds

$$
\begin{gathered}
\left|\frac{\delta}{\delta A_{b}} Q_{k}\left(U_{0}, \eta A, c\right)\right| \leqq 1+2 C_{1}^{\prime} \alpha_{0}+C_{3}|A|<1+2 C_{1}^{\prime} \alpha_{0}+C_{3} \alpha_{1}, \\
\left|\frac{\delta}{\delta A_{b}} C_{k}\left(U_{0}, A, c\right)\right| \leqq C_{3}|A|<C_{3} \alpha_{1} .
\end{gathered}
$$

\section{E. Analyticity Properties of the Averaging Operations}

In this section we will prove some simple analyticity results for the averages. Let us begin with the average $\bar{U}^{k}$. Formally, it is defined for all configurations, but we have good control over it for configurations $U$ satisfying the regularity condition (52). We will prove that $\bar{U}^{k}$ is an analytic function of $U$ on this domain. In fact, we will prove a little bit stronger result. Let us take a configuration $U_{0}$ satisfying (52) and $U=U^{\prime} U_{0}, U^{\prime}=e^{i \eta A^{\prime}},\left|A^{\prime}\right|$ bounded by a small constant $\alpha_{1}$. Such configurations $U$ do not necessarily satisfy (52), so we get a neighborhood of $U_{0}$ which is larger than neighborhoods of $U_{0}$ in the domain. This neighborhood may be also described by the conditions

$$
\left|U-U_{0}\right|=\left|U U_{0}^{-1}-1\right|<\alpha_{1} \eta,
$$

$\alpha_{1}$ is a sufficiently small number.

We will prove that $\bar{U}^{k}={\overline{U^{\prime} U_{0}}}^{k}$ is an analytic function of $A^{\prime}$ and $\bar{U}^{k}\left(\bar{U}_{0}^{k}\right)^{-1}$ is close to 1 ; the difference may be estimated by a constant proportional to $\alpha_{1}$. We have

$$
\begin{aligned}
\bar{U}_{b}^{k}\left(\bar{U}_{0}^{k}\right)_{b}^{-1} & =\left(\overline{U^{\prime} U_{0}}\right)_{b}^{k}\left(\bar{U}_{0}^{k}\right)_{b}^{-1}=\left(\tilde{U}^{\prime}\right)_{b}^{k} \\
& =v_{k}\left(b_{-}\right)\left(\overline{\bar{U}}^{\prime k}\right)_{b} \bar{R}_{0, b}^{k} v_{k}^{-1}\left(b_{+}\right), \quad b \subset \Omega^{(k)},
\end{aligned}
$$

where

$$
v_{k}(x)=\left(\overline{R_{0, x} U^{\prime}}\right)\left(\overline{\bar{R}_{0, x} \overline{\bar{U}}^{\prime}}\right) \cdot \ldots \cdot\left(\overline{\bar{R}_{0, x}^{k-1} \overline{\bar{U}}^{\prime k-1}}\right), \quad x \in \Omega^{(k)} .
$$

From Proposition 4, and especially from (131), we get

$$
\left|\frac{1}{i} \log {\overline{U^{\prime}}}^{\prime j}\right|=\left|Q_{j}\left(U_{0}, \eta A^{\prime}\right)\right|<2 \alpha_{1} L^{j} \eta,
$$

hence

$$
\begin{aligned}
\left|\frac{1}{i} \log \left(\overline{\bar{R}_{0, x}^{j} \overline{\bar{U}}^{\prime j}}\right)\right| & =\left|\sum_{x_{j} \in B(x)} L^{-d} \frac{1}{i} \log \left(\bar{R}_{0, x}^{j} \overline{\bar{U}}^{\prime j}\right)\left(\Gamma_{x, x_{j}}\right)\right| \\
& <8 \alpha_{1} d L^{j+1} \eta e^{2 \alpha_{1} d L^{j+1} \eta}<O(1) \alpha_{1} L^{j+1} \eta, \quad j=0,1, \ldots, k-1
\end{aligned}
$$

and

$$
\left|v_{k}(x)-1\right|<O(1) \alpha_{1} \sum_{j=0}^{k-1} L^{j+1} \eta \leqq O(1) \alpha_{1}
$$

for $\alpha_{1}$ sufficiently small. Moreover, Proposition 4 implies that the functions of $A$ in (159) are analytic. We get the following 
Proposition 6. If $U_{0}$ satisfies (52), then $\overline{U^{\prime} U_{0}}{ }^{k}$ is an analytic function of $A^{\prime}=\frac{1}{i \eta} \log U^{\prime}$ for $A^{\prime}$ with values in the complexified algebra, and satisfying $\left|A^{\prime}\right|<\alpha_{1}$. Moreover, we have a bound

$$
\left|{\overline{U^{\prime} U_{0}}}^{k}\left(\bar{U}_{0}^{k}\right)^{-1}-1\right|<O(1) \alpha_{1} .
$$

Of course, we assume that $\alpha_{0}, \alpha_{1}$ are sufficiently small.

Another analyticity result we will need is an analyticity of $Q_{k}\left(U_{0}, \eta A\right)$ with respect to $U_{0}$. We will understand it in a similar way as for $\bar{U}^{k}$. We take $U^{\prime} U_{0}$ instead of $U_{0}, U^{\prime}=e^{i \eta A^{\prime}},\left|A^{\prime}\right|<\alpha_{1}$, and we consider the function $Q_{k}\left(U^{\prime} U_{0}, \eta A\right)$. We want to prove that it is an analytic function of both variables $A^{\prime}$ and $A$, and that Proposition 4 holds uniformly with respect to $A^{\prime}$.

Let us analyze the proof of Proposition 3 first. The bounds depend on bounds of the quantities $Y_{x}=\frac{1}{i} \log V_{0}\left(\Gamma_{c, x} \cup(-c)\right)$. Previously we had $\left|Y_{x}\right|=O\left(L^{2} \alpha_{0}\right)$, but now we allow complex perturbations $V^{\prime} V_{0}$ of $V_{0}$, and for these we have $\left|Y_{x}\right|=O\left(L^{2} \alpha_{0}+L \alpha_{1}\right)$. Thus Proposition 3 holds unifirmly for $V^{\prime} V_{0}$ instead of $V_{0}$ and with the only change in the inequality (126), where the constant $e^{O(1) L^{2} \alpha_{0}}$ on the right-hand side is replaced by $e^{O(1)\left(L^{2} \alpha_{0}+L \alpha_{1}\right)}$. Similarly we repeat the reasoning connected with Proposition 4, but with $\bar{U}_{0}^{j}$ replaced by $\bar{U}^{\prime} U_{0}{ }^{j}=\widetilde{U}^{j} \bar{U}_{0}^{j}$. Because of the bound (164), we have to replace the factors $e^{O(1) L^{2(j+1)} \eta^{2} \alpha_{0}}$ by $e^{O(1)\left(L^{2(j+1)} \eta^{2} \alpha_{0}+L^{j+1} \eta \alpha_{1}\right)}$, but this change is easily incorporated into the considerations and the estimates. We get the same results as before for $\alpha_{0}, \alpha_{1}$ sufficiently small, uniformly in $A^{\prime}$, and additionally, we get the analyticity of $Q_{k}$ with respect to $A^{\prime}$. Let us formulate these results in

Proposition 7. For $U_{0}$ satisfying (52) and $U^{\prime}=e^{i \eta A^{\prime}},\left|A^{\prime}\right|<\alpha_{1}, \alpha_{0}, \alpha_{1}$ sufficiently small, the function $Q_{k}\left(U^{\prime} U_{0}, \eta A\right)$ is analytic in complex variables $A^{\prime}, A$, and Proposition 4 holds uniformly in $A^{\prime}$.

Similarly, Proposition 5 may be extended to include analyticity and uniformity statements. The formulations are obvious.

\section{F. Averaging Operations for Gauge Transformations}

In the last section of the paper we will study the averaging operations for gauge transformations, given by (61), (78)-(80). A natural analog of the regularity condition (52) would be the condition

$$
\left|\left(\partial_{U_{0}} u\right)(b)\right|=\left|R\left(U_{0, b}\right) u\left(b_{+}\right)-u\left(b_{-}\right)\right|<\alpha_{0} \eta, \quad b \subset \Omega .
$$

We will consider functions $u$ satisfying this condition, but we have to consider also the functions $u$ given by the formulas (104)-(106). They appear naturally in our considerations and generally they do not satisfy the regularity condition (165), but they satisfy other conditions following from (107), (108) if the configuration $U_{1}$ is small, i.e., $\left|U_{1}-1\right|<\alpha_{1} \eta, \alpha_{1}$ small. We define: 
$\Lambda_{k}\left(U_{0}, \alpha_{3}\right)$ is a set of gauge transformations $u$ defined on $\Omega$, and satisfying the conditions

$$
\begin{gathered}
\left|\left(\overline{R_{0} u^{j}}\right)\left(x_{j}\right)-1\right|<\alpha_{3}, x_{j} \in \Omega^{(j)}, \quad j=0,1, \ldots, k, \\
\left|\left(\overline{R_{0} u^{j}}\right)^{-1}\left(x_{j+1}\right)\left(\bar{R}_{0, x_{j+1}}^{j} \overline{R_{0} u^{j}}\right)\left(x_{j}\right)-1\right|<\alpha_{3} L^{j+1} \eta, \\
x_{j+1} \in \Omega^{(j+1)}, x_{j} \in B\left(x_{j+1}\right), \quad j=0,1, \ldots, k-1 .
\end{gathered}
$$

From this it is obvious that for $u \in \Lambda_{k}\left(U_{0}, \alpha_{3}\right)$ and $\alpha_{3}$ small, all operations needed to define ${\overline{R_{0}}}^{k}$ are done always in a case where proper expressions are small. More exactly, we have to calculate a logarithm of the expression in (167) and this expression is small.

We need to consider a product of two gauge transformations satisfying (166), (167), so we would like to know that it also satisfies similar conditions. Let us consider at first the following situation: we have functions $v, v_{1}, v_{2}$ defined on a lattice $\Omega^{\prime}$ and a gauge field configuration $V_{0}$, and we assume that $v(x)=v_{1}(x) v_{2}(x) e^{i r(x)},|r(x)|<c_{1},\left|v_{i}^{-1}(y)\left(R_{0, y} v_{i}\right)(x)-1\right|<c_{2}, \quad i=1,2, \quad x \in B(y)$, $y \in \Omega^{\prime(1)}$. We will find relations between $\overline{R_{0} v}$ and $\overline{R_{0} v_{1}}, \overline{R_{0} v_{2}}$, and bounds satisfied by $\overline{R_{0} v}$. We have

$$
\begin{aligned}
v^{-1}(y)\left(R_{0, y} v\right)(x)= & e^{-i r(y)} v_{2}^{-1}(y) v_{1}^{-1}(y)\left(R_{0, y} v_{1}\right)(x) \\
& \cdot\left(R_{0, y} v_{2}\right)(x) e^{i\left(R_{0, y} r\right)(x)} \\
= & e^{-i r(y)} R\left(v_{2}^{-1}(y)\right)\left[v_{1}^{-1}(y)\left(R_{0, y} v_{1}\right)(x)\right] \\
& \cdot v_{2}^{-1}(y)\left(R_{0, y} v_{2}\right)(x) e^{i\left(R_{0, y} r\right)(x)} \\
= & \exp \left[-i r(y)+i R\left(v_{2}^{-1}(y)\right) \frac{1}{i} \log v_{1}^{-1}(y)\left(R_{0, y} v_{1}\right)(x)\right. \\
& \left.+i \frac{1}{i} \log v_{2}^{-1}(y)\left(R_{0, y} v_{2}\right)(x)+i\left(R_{0, y} r\right)(x)+O\left(\left(c_{1}+c_{2}\right)^{2}\right)\right],
\end{aligned}
$$

hence

$$
\begin{aligned}
\left(\overline{R_{0} v}\right)(y)= & v(y) \exp \left[i \sum_{x \in B(y)} L^{-d} \log v^{-1}(y)\left(R_{0, y} v\right)(x)\right]=v_{1}(y) v_{2}(y) \\
& \cdot \exp \left[i r(y)+i \sum_{x \in B(y)} L^{-d} \log v^{-1}(y)\left(R_{0, y} v\right)(x)+O\left(\left(c_{1}+c_{2}\right)^{2}\right)\right] \\
= & v_{1}(y) v_{2}(y) \exp \left[i \sum_{x \in B(y)} L^{-d} R\left(v_{2}^{-1}(y)\right) \frac{1}{i} \log v_{1}^{-1}(y)\left(R_{0, y} v_{1}\right)(x)\right. \\
& +i \sum_{x \in B(y)} L^{-d} \frac{1}{i} \log v_{2}^{-1}(y)\left(R_{0, y} v_{2}\right)(x) \\
& \left.+i \sum_{x \in B(y)} L^{-d}\left(R_{0, y} r\right)(x)+O\left(\left(c_{1}+c_{2}\right)^{2}\right)\right] \\
= & v_{1}(y) v_{2}(y) R\left(v_{2}^{-1}(y)\right) \exp \left[i \sum_{x \in B(y)} L^{-d} \frac{1}{i} \log v_{1}^{-1}(y)\left(R_{0, y} v_{1}\right)(x)\right] \\
& \cdot \exp \left[i \sum_{x \in B(y)} L^{-d} \frac{1}{i} \log v_{2}^{-1}(y)\left(R_{0, y} v_{2}\right)(x)\right] \\
& \cdot \exp \left[i \sum_{x \in B(y)} L^{-d}\left(R_{0, y} r\right)(x)+O\left(\left(c_{1}+c_{2}\right)^{2}\right)\right] \\
= & \left(\overline{R_{0} v_{1}}\right)(y)\left(\overline{R_{0} v_{2}}\right)(y) e^{i \bar{r}(y)},|\bar{r}(y)|<c_{1}+O\left(\left(c_{1}+c_{2}\right)^{2}\right) .
\end{aligned}
$$


Let us denote the constant in the above bound by $C_{3}$, so we have

$$
\left(\overline{R_{0} v}\right)(y)=\left(\overline{R_{0} v_{1}}\right)(y)\left(\overline{R_{0} v_{2}}\right)(y) e^{i \bar{r}(y)},|\bar{r}(y)|<c_{1}+C_{3}\left(c_{1}+c_{2}\right)^{2}, y \in \Omega^{\prime(1)} .
$$

Now let us take two gauge transformations $u_{1}, u_{2}$ satisfying (166), (167). Applying the above result to $u=u_{1} u_{2}$, we get

$$
\left(\overline{R_{0} u}\right)\left(x_{1}\right)=\left(\overline{R_{0} u_{1}}\right)\left(x_{1}\right)\left(\overline{R_{0} u_{2}}\right)\left(x_{1}\right) e^{i r_{1}\left(x_{1}\right)},\left|r_{1}\left(x_{1}\right)\right|<C_{3}\left(\alpha_{3} L \eta\right)^{2}
$$

for $x_{1} \in \Omega^{(1)}$. Next applying it to $\overline{R_{0} u}, \overline{R_{0} u_{1}}, \overline{R_{0} u_{2}}$, we get

$$
\begin{aligned}
\left({\overline{R_{0} u^{2}}}^{2}\left(x_{2}\right)\right. & =\left({\overline{R_{0} u_{1}}}^{2}\right)\left(x_{2}\right)\left({\overline{R_{0} u_{2}}}^{2}\right)\left(x_{2}\right) e^{i r_{2}\left(x_{2}\right)}, \\
\left|r_{2}\left(x_{2}\right)\right| & <C_{3}\left(\alpha_{3} L \eta\right)^{2}+C_{3}\left(C_{3}\left(\alpha_{3} L \eta\right)^{2}+\alpha_{3} L^{2} \eta\right)^{2} \\
& \leqq C_{3}\left(\alpha_{3} L^{2} \eta\right)^{2}\left[1+L^{-2}\left(1+C_{3} \alpha_{3}\right)^{2}\right]
\end{aligned}
$$

for $x_{2} \in \Omega^{(2)}$. We can prove by an easy induction that

$$
\begin{aligned}
\left(\overline{R_{0} u^{j}}\right)\left(x_{j}\right) & =\left(\overline{R_{0} u_{1}^{j}}\right)\left(x_{j}\right)\left(\overline{R_{0} u_{2}^{j}}\right)\left(x_{j}\right) e^{i r_{j}\left(x_{j}\right)}, \quad x_{j} \in \Omega^{(j)}, \\
\left|r_{j}\left(x_{j}\right)\right| & <C_{3}\left(\alpha_{3} L^{j} \eta\right)^{2}\left[1+L^{-2}\left(1+C_{3} \alpha_{3}\right)^{2}+\ldots+\left(L^{-2}\left(1+C_{3} \alpha_{3}\right)^{2}\right)^{j-1}\right] \\
& <2 C_{3}\left(\alpha_{3} L^{j} \eta\right)^{2}
\end{aligned}
$$

for $\alpha_{3}$ sufficiently small, i.e., such that $L^{-2}\left(1+C_{3} \alpha_{3}\right)^{2} \leqq \frac{1}{2}$. From this it follows that

$$
\begin{aligned}
& \left|\left(\overline{R_{0} u^{j}}\right)\left(x_{j}\right)-1\right|<2 \alpha_{3}+2 C_{3}\left(\alpha_{3} L^{j} \eta\right)^{2} \leqq 2 \alpha_{3}+2 C_{3} \alpha_{3}^{2}, \quad j=0,1, \ldots, k, \\
& \left|\left(\bar{R}_{0} u^{j}\right)^{-1}\left(x_{j+1}\right)\left(\bar{R}_{0, x_{j+1}} \overline{R_{0}} u^{j}\right)\left(x_{j}\right)-1\right|<2 \alpha_{3} L^{j+1} \eta+4 C_{3}\left(\alpha_{3} L^{j} \eta\right)^{2} \\
& \quad<\left(2 \alpha_{3}+2 C_{3} \alpha_{3}^{2}\right) L^{j+1} \eta, \quad j=0,1, \ldots, k-1 .
\end{aligned}
$$

We can formulate these results in

Proposition 8. If $u_{1}, u_{2} \in \Lambda_{k}\left(U_{0}, \alpha_{3}\right)$ and $\alpha_{3}$ is sufficiently small, i.e., $\alpha_{3} \leqq c_{6}$ for some $c_{6}$, then $u=u_{1} u_{2} \in \Lambda_{k}\left(U_{0}, 2 \alpha_{3}+2 C_{3} \alpha_{3}^{2}\right)$ and we have (173).

We will need to consider regular configurations $u^{\prime}$ in the sense that the following conditions are satisfied:

$$
\begin{gathered}
\left|u^{\prime}(x)-1\right|<\alpha_{4}, \quad x \in \Omega, \\
\left|u^{\prime-1}\left(b_{-}\right) R_{0, b} u^{\prime}\left(b_{+}\right)-1\right|<\alpha_{4} \eta, \quad b \subset \Omega .
\end{gathered}
$$

We would like to know that if $u^{\prime}$ is such a configuration and $u_{1}$ belongs to a class $\Lambda_{k}\left(\alpha_{3}\right)$, then the product $u^{\prime} u_{1}$ belongs to some class $\Lambda_{k}\left(O(1)\left(\alpha_{3}+\alpha_{4}\right)\right)$ also. Because we want some analyticity properties of $\frac{1}{i} \log {\overline{R_{0} u^{\prime} u_{1}}}^{k}$, we will consider configurations $u^{\prime}$ with values in the complexified group $G^{c}$, i.e., $u^{\prime}=e^{i \lambda}$ and $\lambda$ has values in the complexified algebra $\mathfrak{g}^{c}$. We will consider the averages

$$
\tilde{u}^{\prime j}=\overline{R_{0} u^{\prime} u_{1}^{j}}\left(\overline{R_{0} u_{1}^{j}}\right)^{-1} \text {. }
$$

As in the case of averages $\tilde{U}^{\prime j}$, it can be easily seen that they may be defined inductively as

$$
\tilde{u}^{\prime 1}=\tilde{u}^{\prime}=\overline{R_{0} u^{\prime} u_{1}}\left(\overline{R_{0} u_{1}}\right)^{-1}, \tilde{u}^{\prime j+1}=\overline{\bar{R}_{0}^{j} \tilde{u}^{j} \overline{R_{0} u_{1}}{ }^{j}}\left(\overline{\bar{R}_{0}^{j}}{\overline{R_{0} u_{1}}}^{j}\right)^{-1} .
$$


We will prove that the configuration $\tilde{u}^{\prime j}$ for $j \leqq k$ satisfy the regularity conditions (176), (177) on proper scales and with different constants. As usual, we start with a careful analysis of a one-step operation. Let us assume that we have a gauge field configuration $V_{0}$ satisfying the regularity condition $\left|V_{0}(\partial p)-1\right|<\alpha_{0}, p \subset \Omega^{\prime}$, and two gauge transformations $v^{\prime}, v_{1}$ satisfying the conditions

$$
\begin{aligned}
& \left|v^{\prime}-1\right|<\alpha_{4},\left|v^{-1}\left(b_{-}\right) R_{0, b} v^{\prime}\left(b_{+}\right)-1\right|<\alpha_{4}^{\prime}, \\
& \left|v_{1}-1\right|<\alpha_{3},\left|v_{1}^{-1}(y)\left(R_{0} v_{1}\right)(x)-1\right|<L \alpha_{3}^{\prime},
\end{aligned}
$$

$x \in B(y), y \in \Omega^{\prime(1)}$. We assume that the constants are sufficiently small, so that we can apply proper theorems and estimates. We will find a bound for $\tilde{v}^{\prime-1}\left(c_{-}\right) R_{0, c^{\prime}} \tilde{v}^{\prime}\left(c_{+}\right)-1$. We have

$$
\begin{gathered}
\left(\overline{R_{0} v^{\prime} v_{1}}\right)(y)=v^{\prime}(y) v_{1}(y) \\
\cdot \exp \left[i \sum_{x \in B(y)} L^{-d} \frac{1}{i} \log \left(v^{\prime} v_{1}\right)^{-1}(y)\left(R_{0, y} v^{\prime} v_{1}\right)(x)\right], \\
\left(v^{\prime} v_{1}\right)^{-1}(y)\left(R_{0, y} v^{\prime} v_{1}\right)(x)=R\left(v_{1}^{-1}(y)\right)\left[v^{\prime-1}(y)\left(R_{0, y} v^{\prime}\right)(x)\right] \\
\cdot v_{1}^{-1}(y)\left(R_{0, y} v_{1}\right)(x),
\end{gathered}
$$

and $\left|v^{\prime-1}(y)\left(R_{0, y} v^{\prime}\right)(x)-1\right|<\left|\Gamma_{y, x}\right| \alpha_{4}^{\prime} e^{\left|\Gamma_{y, x}\right| \alpha_{4}^{\prime}}=O\left(L \alpha_{4}^{\prime}\right)$ by (180), hence

$$
\begin{gathered}
\frac{1}{i} \log \left(v^{\prime} v_{1}\right)^{-1}(y)\left(R_{0, y} v^{\prime} v_{1}\right)(x)=R\left(v_{1}^{-1}(y)\right) \frac{1}{i} \log v^{-1}(y) \\
\cdot\left(R_{0, y} v^{\prime}\right)(x)+\frac{1}{i} \log v_{1}^{-1}(y)\left(R_{0, y} v_{1}\right)(x)+O\left(L^{2} \alpha_{3}^{\prime} \alpha_{4}^{\prime}\right) .
\end{gathered}
$$

A constant in the bound above is an absolute constant. Using (41) and (181), (183) we get

$$
\begin{aligned}
\tilde{v}^{\prime}(y)= & v^{\prime}(y) v_{1}(y) \exp \left[i \sum_{x \in B(y)} L^{-d} R\left(v_{1}^{-1}(y)\right) \frac{1}{i} \log v^{-1}(y)\right. \\
& \left.\cdot\left(R_{0, y} v^{\prime}\right)(x)+O\left(L^{2} \alpha_{3}^{\prime} \alpha_{4}^{\prime}\right)+\left(L^{2} \alpha_{4}^{\prime 2}\right)\right] v_{1}^{-1}(y) \\
= & v^{\prime}(y) \exp \left[i \sum_{x \in B(y)} L^{-d} \frac{1}{i} \log v^{\prime-1}(y)\left(R_{0, y} v^{\prime}\right)(x)\right. \\
& \left.+O\left(L^{2}\left(\alpha_{3}^{\prime}+\alpha_{4}^{\prime}\right) \alpha_{4}^{\prime}\right)\right] .
\end{aligned}
$$

Let us denote

$$
\begin{aligned}
& V_{b}^{\prime}=v^{\prime-1}\left(b_{-}\right) R_{0, b} v^{\prime}\left(b_{+}\right), \text {then } \quad\left|V_{b}^{\prime}-1\right|<\alpha_{4}^{\prime} e^{\alpha_{4}^{\prime}}, \\
& V_{b}^{\prime}=e^{i A_{b}}, \quad\left|A_{b}\right|<2 \alpha_{4}^{\prime} .
\end{aligned}
$$

Using this we can write

$$
v^{\prime-1}(y)\left(R_{0, y} v^{\prime}\right)(x)=\prod_{b \subset \Gamma_{y, x}} R\left(V_{0}\left(\Gamma_{y, b_{-}}\right)\right) V_{b}^{\prime}=\left(R_{0, y} V\right)\left(\Gamma_{y, x}\right),
$$


and we have

$$
\frac{1}{i} \log \left(R_{0, y} V^{\prime}\right)\left(\Gamma_{y, x}\right)=\left(R_{0, y} A\right)\left(\Gamma_{y, x}\right)+O\left(\left(L \alpha_{4}^{\prime}\right)^{2}\right) .
$$

Now using this and the representation (184), we have

$$
\begin{aligned}
\tilde{v}^{\prime-1}\left(c_{-}\right) R_{0, c} \tilde{v}^{\prime}\left(c_{+}\right)= & \exp \left[-i \sum_{x \in B\left(c_{-}\right)} L^{-d}\left(R_{0, c_{-}} A\right)\left(\Gamma_{c_{-}, x}\right)\right. \\
& \left.+O\left(L^{2}\left(\alpha_{3}^{\prime}+\alpha_{4}^{\prime}\right) \alpha_{4}^{\prime}\right)\right] v^{\prime-1}\left(c_{-}\right) \bar{R}_{0, c} v^{\prime}\left(c_{+}\right) \\
& \cdot \exp \left[i \sum_{x^{\prime} \in B\left(c_{+}\right)} L^{-d}\left(\bar{R}_{0, c} R_{0, c_{+}} A\right)\left(\Gamma_{c_{+}, x^{\prime}}\right)+O\left(L^{2}\left(\alpha_{3}^{\prime}+\alpha_{4}^{\prime}\right) \alpha_{4}^{\prime}\right)\right] .
\end{aligned}
$$

Let us recall that

$$
\bar{V}_{0, c}=\exp \left[i \sum_{x \in B(c-)} L^{-d} \frac{1}{i} \log V_{0}\left(\Gamma_{c, x} \cup(-c)\right)\right] V_{0}(c)=e^{i O\left(L^{2} \alpha_{0}\right)} V_{0}(c),
$$

hence

$$
\begin{aligned}
\bar{R}_{0, c} v^{\prime}\left(c_{+}\right) & =R\left(V_{0}(c)\right) v^{\prime}\left(c_{+}\right)+O\left(L^{2} \alpha_{0}\left|v^{\prime}\left(c_{+}\right)-1\right|\right) \\
& =R\left(V_{0}(c)\right) v^{\prime}\left(c_{+}\right)+O\left(L^{2} \alpha_{0} \alpha_{4}\right) .
\end{aligned}
$$

Similarly,

$$
\left(\bar{R}_{0, c} R_{0, c_{+}} A\right)\left(\Gamma_{c_{+}, x^{\prime}}\right)=\left(R\left(V_{0}(c)\right) R_{0, c_{+}} A\right)\left(\Gamma_{c_{+}, x^{\prime}}\right)+O\left(L^{2} \alpha_{0} \alpha_{4}^{\prime}\right) .
$$

Further we can write

$$
\begin{aligned}
v^{\prime-1}\left(c_{-}\right) R\left(V_{0}(c)\right) v^{\prime}\left(c_{+}\right) & =\left(R_{0, c_{-}} V^{\prime}\right)(c) \\
& =1+i\left(R_{0, c_{-}} A\right)(c)+O\left(\left(L \alpha_{4}^{\prime}\right)^{2}\right),
\end{aligned}
$$

and this implies the following representation

$$
\begin{aligned}
\tilde{v}^{\prime-1}\left(c_{-}\right) \bar{R}_{0, c^{\prime}} \tilde{v}^{\prime}\left(c_{+}\right)-1= & -i \sum_{x \in B\left(c_{-}\right)} L^{-d}\left(R_{0, c_{-}} A\right)\left(\Gamma_{c_{-}, x}\right) \\
& +i\left(R_{0, c_{-}} A\right)(c)+i \sum_{x^{\prime} \in B\left(c_{+}\right)} L^{-d}\left(R\left(V_{0}(c)\right) R_{0, c_{+}} A\right)\left(\Gamma_{c_{+}, x^{\prime}}\right) \\
& +O\left(L^{2}\left(\alpha_{0} \alpha_{4}+\alpha_{0} \alpha_{4}^{\prime}+\alpha_{3}^{\prime} \alpha_{4}^{\prime}+\alpha_{4}^{\prime 2}\right)\right) .
\end{aligned}
$$

We will transform the linear terms in $A$ on the right-hand side of (189). Let us notice that by the definition (185) of $A$ and by the identity $\log v^{-1}=-\log v$ we have

$$
\begin{aligned}
-A_{b} & =-\frac{1}{i} \log u^{\prime-1}\left(b_{-}\right) R_{0, b} u^{\prime}\left(b_{+}\right)=\frac{1}{i} \log \left(R_{0, b} u^{\prime-1}\left(b_{+}\right)\right) u^{\prime}\left(b_{-}\right) \\
& =R_{0, b} \frac{1}{i} \log u^{\prime-1}\left(b_{+}\right) R_{0,-b} u^{\prime}\left(b_{-}\right) \\
& =R_{0, b} \frac{1}{i} \log u^{\prime-1}\left((-b)_{-}\right) R_{0,-b} u^{\prime}\left((-b)_{+}\right),
\end{aligned}
$$


hence

$$
-A_{b}=R_{0, b} A_{-b} .
$$

For the term $\left(R_{0, c_{-}} A\right)(c)$, we have for $x \in B\left(c_{-}\right)$,

$$
\begin{aligned}
\left(R_{0, c_{-}} A\right)(c) & =\sum_{b \subset c} R\left(V_{0}\left(\left[c_{-}, b_{-}\right]\right)\right) A_{b}=-\sum_{b \subset c} R\left(V_{0}\left(\left[c_{-}, b_{+}\right]\right)\right) A_{-b} \\
& =-\sum_{b c-c} R\left(V_{0}\left(c \cup\left(-\Gamma_{c, x}\right)\right)\right) R\left(V_{0}\left(\Gamma_{c, x} \cup\left[c_{+}, b_{-}\right]\right)\right) A_{b} \\
& =-\sum_{b \subset-c} R\left(V_{0}\left(\Gamma_{c, x} \cup\left[c_{+}, b_{-}\right]\right)\right) A_{b}+O\left(L^{2} \alpha_{0} \alpha_{4}^{\prime}\right) ;
\end{aligned}
$$

similarly for the third term on the right-hand side of (189). Taking this into account, we have

$$
\begin{aligned}
\tilde{v}^{\prime-1}\left(c_{-}\right) \bar{R}_{0, c^{\prime}} \tilde{v}^{\prime}\left(c_{+}\right)-1= & i \sum_{x \in B\left(c_{-}\right)} L^{-d}\left(R_{0, c_{-}} A\right)\left(\left[x, x^{\prime}\right]\right) \\
& -i \sum_{x \in B\left(c_{-}\right)} L^{-d}\left(R_{0, c_{-}} A\right)\left(\Gamma_{c, x} \cup(-c)\right) \\
& +O\left(L^{2}\left(\alpha_{0} \alpha_{4}+\alpha_{0} \alpha_{4}^{\prime}+\alpha_{3}^{\prime} \alpha_{4}^{\prime}+\alpha_{4}^{\prime 2}\right)\right) .
\end{aligned}
$$

Let us now estimate the terms $\left(R_{0, c_{-}} A\right)\left(\Gamma_{c, x} \cup(-c)\right)$. They almost vanish because by the definition (185) $A$ is almost equal to the derivative of $\lambda=\frac{1}{i} \log v^{\prime}$. We will prove in fact that they are small. Similarly, as in (187) we have

$$
\left(R_{0, c_{-}} A\right)\left(\Gamma_{c, x} \cup(-c)\right)=\frac{1}{i} \log \left(R_{0, c_{-}} V\right)\left(\Gamma_{c, x} \cup(-c)\right)+O\left(\left(L \alpha_{4}^{\prime}\right)^{2}\right),
$$

and

$$
\begin{aligned}
\left(R_{0, c_{-}} V^{\prime}\right)\left(\Gamma_{c, x} \cup(-c)\right) & =v^{-1}\left(c_{-}\right) R\left(V_{0}\left(\Gamma_{c, x} \cup(-c)\right)\right) v^{\prime}\left(c_{-}\right) \\
& =1+v^{-1}\left(c_{-}\right)\left[R\left(V_{0}\left(\Gamma_{c, x} \cup(-c)\right)\right)-1\right]\left(v^{\prime}\left(c_{-}\right)-1\right) \\
& =1+O\left(L^{2} \alpha_{0} \alpha_{4}\right),
\end{aligned}
$$

hence

$$
\left(R_{0, c_{-}} A\right)\left(\Gamma_{c, x} \cup(-c)\right)=O\left(L^{2}\left(\alpha_{0} \alpha_{4}+\alpha_{4}^{\prime 2}\right)\right) .
$$

This gives us finally

$$
\begin{aligned}
\tilde{v}^{\prime-1}\left(c_{-}\right) \bar{R}_{0, c} \tilde{v}^{\prime}\left(c_{+}\right)-1= & i \sum_{x \in B(c)} L^{-d}\left(R_{0, c-} A\right)\left(\left[x, x^{\prime}\right]\right) \\
& +O\left(L^{2}\left(\alpha_{0} \alpha_{4}+\alpha_{0} \alpha_{4}^{\prime}+\alpha_{3}^{\prime} \alpha_{4}^{\prime}+\alpha_{4}^{\prime 2}\right)\right) .
\end{aligned}
$$

From this we easily get a bound if we notice that

$$
A_{b}=\frac{1}{i}\left(v^{\prime-1}\left(b_{-}\right) R_{0, b} v^{\prime}\left(b_{+}\right)-1\right)+O\left(\alpha_{4}^{\prime 2}\right) .
$$

This implies $\left|A_{b}\right|<\alpha_{4}^{\prime}+O\left(\alpha_{4}^{\prime 2}\right)$, and

$$
\left|\tilde{v}^{\prime-1}\left(c_{-}\right) \bar{R}_{0, c^{\prime}} \tilde{v}^{\prime}\left(c_{+}\right)-1\right|<L \alpha_{4}^{\prime}+O\left(L^{2}\left(\alpha_{0} \alpha_{4}+\alpha_{0} \alpha_{4}^{\prime}+\alpha_{3}^{\prime} \alpha_{4}^{\prime}+\alpha_{4}^{\prime 2}\right)\right) .
$$


From representation (184) we obtain also

$$
\left|\tilde{v}^{\prime}(y)-1\right|<\alpha_{4}+O\left(L \alpha_{4}^{\prime}\right)+O\left(L^{2}\left(\alpha_{3}^{\prime}+\alpha_{4}^{\prime}\right) \alpha_{4}^{\prime}\right) \leqq \alpha_{4}+O\left(L \alpha_{4}^{\prime}\right) .
$$

Let us formulate these results in

Proposition 9. There exist positive constants $C_{4}^{\prime}, C_{5}^{\prime}, c_{6}^{\prime}$ such that for arbitrary functions $V_{0}, v^{\prime}, v_{1}$ satisfying (180) with $\alpha_{0}, \alpha_{3}, \alpha_{3}^{\prime}, \alpha_{4}, \alpha_{4}^{\prime} \leqq c_{6}^{\prime}$, the following bounds hold:

$$
\begin{gathered}
\left|\tilde{v}^{\prime-1}\left(c_{-}\right) \bar{R}_{0, c} \tilde{v}^{\prime}\left(c_{+}\right)-1\right|<L \alpha_{4}^{\prime}+C_{4}^{\prime} L^{2}\left(\alpha_{0} \alpha_{4}+\alpha_{3}^{\prime} \alpha_{4}^{\prime}+\alpha_{4}^{\prime 2}\right), \\
\left|\tilde{v}^{\prime}(y)-1\right|<\alpha_{4}+C_{5}^{\prime} L \alpha_{4}^{\prime} .
\end{gathered}
$$

In these bounds we have assumed that $\alpha_{4}^{\prime}=O\left(\alpha_{4}\right)$, which will always be true here and in forthcoming papers.

Let us apply this result to configurations $U_{0}, u^{\prime}, u_{1}$ many times. We assume that $U_{0}$ satisfies (52), $u$ satisfies (176), (177), and $u_{1}$ satisfies (166), (167). When we apply it the first time, we get bounds

$$
\begin{gathered}
\left|\tilde{u}^{\prime-1}\left(c_{-}\right) \bar{R}_{0, c} \tilde{u}^{\prime}\left(c_{+}\right)-1\right|<\alpha_{4} L \eta+C_{4}^{\prime}\left(\alpha_{0} \alpha_{4}+\alpha_{3} \alpha_{4}+\alpha_{4}^{2}\right)(L \eta)^{2}, \\
\left|\tilde{u}^{\prime}(y)-1\right|<\alpha_{4}+C_{5}^{\prime} \alpha_{4} L \eta .
\end{gathered}
$$

Let us denote $\beta=\alpha_{0} \alpha_{4}+\alpha_{3} \alpha_{4}+\alpha_{4}^{2}, C_{5}=1+4 C_{5}^{\prime}, C_{4}=8 C_{4}^{\prime} C_{5}$. We will prove by induction that

$$
\begin{gathered}
\left|\left(\tilde{u}^{j}\right)^{-1}\left(c_{-}\right) \bar{R}_{0, c_{u}^{j}} \tilde{u}^{\prime j}\left(c_{+}\right)-1\right|<\alpha_{4} L^{j} \eta+C_{4} \beta\left(L^{j} \eta\right)^{2}, \quad c \subset \Omega^{(j)}, \\
\left|\tilde{u}^{\prime j}(y)-1\right|<\alpha_{4}+2 C_{5}^{\prime} \alpha_{4} L \eta+\ldots+2 C_{5}^{\prime} \alpha_{4} L^{j} \eta, \quad y \in \Omega^{(j)} .
\end{gathered}
$$

Let us notice that

$$
\alpha_{4} L^{j} \eta+C_{4} \beta\left(L^{j} \eta\right)^{2} \leqq \alpha_{4} L^{j} \eta\left(1+C_{4}\left(\alpha_{0}+\alpha_{3}+\alpha_{4}\right)\right) \leqq 2 \alpha_{4} L^{j} \eta \leqq 2 \alpha_{4}
$$

for $\alpha_{0}, \alpha_{3}, \alpha_{4}$ sufficiently small, so the condition $\alpha_{4}^{\prime}=O\left(\alpha_{4}\right)$ mentioned above is satisfied indeed. The inequalities (203), (204) hold for $j=0,1$. We assume them for some $j$ and we will prove them for $j+1$. We apply Proposition 9 with $V_{0}=\bar{U}_{0}^{j}$, $v^{\prime}=\tilde{u}^{j}{ }^{j}, v_{1}={\overline{R_{0} u_{1}}}^{j}$. We have $\alpha_{0}$ replaced by $2 \alpha_{0}\left(L^{j} \eta\right)^{2}, \alpha_{3}^{\prime}$ replaced by $\alpha_{3} L^{j} \eta, \alpha_{4}$ replaced by the right-hand side of (204), which can be bounded by $\alpha_{4}\left(1+4 C_{5}^{\prime}\right)=C_{5} \alpha_{4}$, and $\alpha_{4}^{\prime}$ replaced by the right-hand side of (203), which can be bounded by $2 \alpha_{4}$. The conditions of the proposition are satisfied if, e.g., $2 \alpha_{0}, \alpha_{3}$, $C_{5} \alpha_{4}, 2 \alpha_{4} \leqq c_{6}^{\prime}$, so we can apply it and we get

$$
\begin{aligned}
& \left|\left(\tilde{u}^{j+1}\right)^{-1}\left(c_{-}\right) \bar{R}_{0, c}^{j+1} \tilde{u}^{j+1}\left(c_{+}\right)-1\right|<\alpha_{4} L^{j+1} \eta+L C_{4} \beta\left(L^{j} \eta\right)^{2} \\
& \quad+C_{4}^{\prime}\left(2 \alpha_{0} C_{5} \alpha_{4}+\alpha_{3} 2 \alpha_{4}+4 \alpha_{4}^{2}\right)\left(L^{j+1} \eta\right)^{2} \\
& <\alpha_{4} L^{j+1} \eta+C_{4} \beta\left(L^{j+1} \eta\right)^{2}\left[L^{-1}+\frac{C_{4}^{\prime} 4 C_{5}}{C_{4}}\right] \\
& \leqq \alpha_{4} L^{j+1} \eta+C_{4} \beta\left(L^{j+1} \eta\right)^{2}, \\
& \left|\tilde{u}^{j+1}(y)-1\right|<\alpha_{4}+2 C_{5}^{\prime} \alpha_{4} L \eta+\ldots+2 C_{5}^{\prime} \alpha_{4} L^{j} \eta+2 C_{5}^{\prime} \alpha_{4} L^{j+1} \eta .
\end{aligned}
$$

Thus we have proved the following 
Proposition 10. There exist positive constants $C_{4}, C_{5}, c_{6}$ such that for arbitrary configurations $U_{0}, u^{\prime}, u_{1}$ satisfying (52), (176), (177), (166), (167) with $\alpha_{0}, \alpha_{3}, \alpha_{4} \leqq c_{6}$ the bounds (203), (204) hold for $j \leqq k$.

This result implies in particular that the configuration $u^{\prime}$ belongs to the class $\Lambda_{k}\left(C_{5} \alpha_{4}\right)$. The assumptions (176), (177) can be reformulated in terms of the functions $\lambda=\frac{1}{i} \log u^{\prime}$. If we assume

$$
\begin{gathered}
\left|\left(D_{U_{0}}^{\eta} \lambda\right)(b)\right|<\alpha_{4}, \quad|\lambda(x)|<\alpha_{4}, \quad \lambda(x) \in \mathfrak{g}^{c}, \\
\alpha_{4} \text { sufficiently small, }
\end{gathered}
$$

then assumptions (176), (177) are satisfied with a constant $4 \alpha_{4}$ instead of $\alpha_{4}$. It is obvious from the definition of the averaging operations that $\tilde{u}^{\prime j}$ are analytic functions of $\lambda$, and

$$
Q_{j}^{\prime}\left(u_{1}, \lambda\right)=\frac{1}{i} \log \tilde{u}^{\prime j}, \quad j \leqq k,
$$

are analytic functions of $\lambda$ also. We want to calculate a linear term in an expansion of this function. In fact, we will be satisfied with a good approximation of this term. From (184), (187), we have for $v^{\prime}=e^{i \lambda}$

$$
\tilde{v}^{\prime}(y)=\exp \left[i \lambda(y)+i \sum_{x \in B(y)} L^{-d}\left(R_{0, y} A\right)\left(\Gamma_{y, x}\right)+O\left(\alpha_{4} L \alpha_{4}^{\prime}\right)+O\left(L^{2}\left(\alpha_{3}^{\prime}+\alpha_{4}^{\prime}\right) \alpha_{4}^{\prime}\right)\right] .
$$

By definition of $A$,

$$
\begin{aligned}
A_{b} & =\frac{1}{i} \log e^{-i \lambda\left(b_{-}\right)} e^{i R_{0, b} \lambda\left(b_{+}\right)} \\
& =\frac{1}{i} \log e^{-i \lambda(b-)} e^{i \lambda\left(b_{-}\right)+i\left(D_{V_{0}} \lambda\right)(b)} \\
& =\left(D_{V_{0}} \lambda\right)(b)+O\left(\alpha_{4} \alpha_{4}^{\prime}+\alpha_{4}^{\prime 2}\right),
\end{aligned}
$$

hence

$$
\frac{1}{i} \log \tilde{v}^{\prime}(y)=\sum_{x \in B(y)} L^{-d}\left(R_{0, y} \lambda\right)(x)+O\left(\alpha_{4} L \alpha_{4}^{\prime}+L^{2} \alpha_{3}^{\prime} \alpha_{4}^{\prime}+L^{2} \alpha_{4}^{\prime 2}\right) .
$$

By (179) the function $Q_{j}^{\prime}\left(u_{1}, \lambda\right)$ is a composition of one-step functions and from the above formula we can easily see that

$$
\begin{aligned}
Q_{j}^{\prime}\left(u_{1}, \lambda, y\right)= & \sum_{x \in B^{j}(y)} L^{-j d} R\left(U_{0}\left(\Gamma_{y, x}^{(j)}\right)\right) \lambda(x)+\sum_{l=0}^{j-1} \\
& \cdot O\left(C_{5} \alpha_{4} 2 \alpha_{4} L^{l} \eta+\alpha_{3} 2 \alpha_{4}\left(L^{l} \eta\right)^{2}+4 \alpha_{4}^{2}\left(L^{l} \eta\right)^{2}\right),
\end{aligned}
$$

hence

$$
\begin{gathered}
Q_{j}^{\prime}\left(u_{1}, \lambda, y\right)=\left(Q_{j}^{\prime} \lambda\right)(y)+C_{j}^{\prime}\left(u_{1}, \lambda, y\right), \\
\left|C_{j}^{\prime}\left(u_{1}, \lambda, y\right)\right|=O\left(\left(\alpha_{3} \alpha_{4}+\alpha_{4}^{2}\right) L^{j} \eta\right) .
\end{gathered}
$$




\section{References}

1. Balaban, T.: (Higgs) ${ }_{2,3}$ quantum fields in a finite volume. I. A lower bound. Commun. Math. Phys. 85, 603-636 (1982)

2. Balaban, T.: Propagators and renormalization transformations for lattice gauge theories. I. Commun. Math. Phys. 95, 17-40 (1984)

3. Brydges, D.C., Fröhlich, J., Seiler, E.: On the construction of quantized gauge fields. I. General results. Ann. Phys. 121, 227-284 (1979)

4. Kadanoff, L.P.: Notes on Migdal's recursion formulas. Ann. Phys. 100, 359-394 (1976)

5. Kadanoff, L.P.: The application of renormalization group techniques to quarks and strings. Rev. Mod. Phys. 49, 267-296 (1977)

6. Osterwalder, K., Seiler, E.: Gauge field theories on a lattice. Ann. Phys. 110, 440-471 (1978)

7. Varadarajan, V.S.: Lie groups, Lie algebras and their representations. New York: PrenticeHall 1974

8. Wilson, K.G.: Confinement of quarks. Phys. Rev. D10, 2445-2459 (1974)

9. Wilson, K.G.: Quantum chromodynamics on a lattice. In: Quantum field theory and statistical mechanics, Cargèse 1976, pp. 143-172. Lévy, M., Mitter, P., eds. New York: Plenum Press 1977

10. Wilson, K.G.: Monte Carlo calculations for the lattice gauge theory. In: Recent developments in gauge theories. pp. 363-402. 't Hooft, G., Itzykson, C., Jaffe, A., Lehmann, M., Mitter, P.K., Singer, I.M., Stora, R., eds. New York: Plenum Press 1980

Communicated by A. Jaffe

Received January 12, 1984; in revised form September 4, 1984 
